\title{
HUMAN MEIOSIS VIII. CHROMOSOME PAIRING AND FORMATION OF THE SYNAPTONEMAL COMPLEX IN OOCYTES
}

\author{
by
}

\author{
MAJA BOJKO
}

\author{
Department of Physiology, Carlsberg Laboratory, \\ Gamle Carlsberg Vej 10, DK-2500, Copenhagen Valby
}

Keywords: Human female meiosis, nucleoli, interlocking, chromosome breakage, nonhomologous pairing, recombination nodules.

\begin{abstract}
Human oocytes from three foetal ovaries have been investigated by serial sectioning and three dimensional reconstruction of nuclei from electron micrographs. Analysis of three leptotene-early zygotene, five mid zygotene and ten late zygotene oocytes including complete reconstruction of five zygotene nuclei have led to the following observations and conclusions. 1) Formation of the synaptonemal complex involves, as in other organisms, the organization of a lateral component along each chromosome, attachment of the telomere regions to the inner membrane of the nuclear envelope at leptotene, redistribution of the attached telomeres during the formation of a chromosome bouquet at early zygotene and thereafter progressive chromosome pairing with the synaptonemal complex. 2) Chromosome and bivalent interlockings as well as breaks were common at mid zygotene, but absent when pairing and synaptonemal complex formation were almost complete. Most of the breaks had occurred in the vicinity of presumptive interlockings and appear to be instrumental in the resolution of interlockings. 3) Nonhomologous pairing involving short regions of the complement were observed in two of the mid zygotene nuclei, thus resulting in associations of three or more chromosomes. 4) Twelve of the 23 bivalents can be identified by relative lengths, centromere indices and the structural markers on chromosomes 1, 6, 9, 13-15 and 21-22. 5) The length of the lateral component complement exceeds that of the spermatocytes by a factor of two. 6) On an average 58 recombination nodules was observed per nucleus at mid zygotene $(61 \%$ of the chromosome complement paired) and 70 at late zygotene ( $98 \%$ of the chromosome complement paired). Thus in spite of the twofold longer synaptonemal complexes in the oocytes, the number of nodules is the same in both sexes.
\end{abstract}

\section{INTRODUCTION}

Meiotic nondisjunction is generally considered to be a major cause of spontaneous abortions in humans (4), and results in cases of liveborn progeny in physical and mental defects. Although a substantial amount of unbalanced embryos can be attributed to meiotic nondisjunction in the male, the investigated cases have shown that supernumerary chromosomes in most cases originate from a failure during female meiosis (23). 
Attempts to elucidate this sex dependent difference require a detailed knowledge of the normal course of meiosis in both sexes. Ultrastructural investigations of meiotic chromosomes have provided detailed information on the course of meiosis in higher organisms (see 8, 22, $29,30,33$ for reviews). Using the approach of serial sectioning and three dimensional reconstruction, the processes of chromosome pairing and synaptonemal complex formation, chiasma formation and disjunction have been characterized in a number of organisms (e.g. 7, 11, 12, $15,17,26,27,35)$ including the human male (13, $18,19,20,28)$. The available corresponding information on human female meiosis is neither adequate for a comparison with spermatogenesis nor as a basis for speculations concerning the factors responsible for the high level of segregation failure.

The difference between the temporal courses of the male and female meiosis is, however, considerable and may constitute one of the factors responsible for the high frequency of female nondisjunction. Spermatogenesis in males commences at puberty and continues throughout life, whereas all meiocytes in the female progress through the early stages of prophase during a period of the foetal life. At 9-10 weeks postconception, primordial germ cells in the foetal ovary undergo mitotic divisions resulting in the production of many thousands of so-called stem cells of which those located in the ovarian cortex become the oogonia (34). The last mitosis is followed by premeiotic DNA replication and at 1213 weeks postconception meiosis commences (21). During the first half period of pregnancy, the number of oogonia and primary oocytes increases until a maximum of almost seven million cells in both ovaries is reached at the 5th month (1). During the last part of pregnancy, an increasing number of the oocytes again degenerate and become engulfed by granulosa cells $(2,9,10)$ leaving, at the time of birth, only about two million oocytes in the two ovaries (1). In the course of the foetal life, meiotic development reaches diplotene, at which stage the oocytes are arrested, for perhaps up to fifty years, until diakinesis followed by the first meiotic division and prometaphase II ensue a few hours before ovulation.

The aim of the present paper is to describe the fine structure of human oocytes in the first stages of meiotic prophase from leptotene to late zygotene, especially focusing on chromosome pairing and synaptonemal complex formation and to compare the observations with those available on male meiosis $(13,18,19,20,28)$.

\section{MATERIALS AND METHODS}

\subsection{Materials}

Foetal ovarian biopsies were kindly provided by Prof. F. Bierring, Winslow Institute of Human Anatomy, University of Odense and Dr. J. GRINDSTED, General Surgery Dept., Herlev Amtsygehus, Copenhagen. The material consists of ovarian tissue from three foetuses (A, B and C) obtained by hysterotomy, the pregnancies in all three cases being terminated for social and psychological indications. Other ovaries, obtained from prostaglandin induced abortions, were not useable as the prolonged hormone treatment caused severe autolysis of the foetal ovary.

The ages of the foetuses were determinated on the basis of their length, rather than on the menstrual history and were estimated to be 16-17 (A), 22-24 (B) and 24-26 (C) weeks postconception. All three ovaries appeared histologically normal, and did not reveal structural differences. The mother of foetus B had been treated with the cytostatic drug imuran, a treatment which as far as can be judged had no effect on the normal progression of meiosis in the ovary.

In this study, ten zygotene nuclei from the three cases were serially sectioned, of which five (four from foetus $\mathrm{B}$ and one from foetus $\mathrm{C}$ ) were reconstructed completely. In addition, three leptotene - early zygotene nuclei, one from each foetus, were serially sectioned and partially reconstructed.

\subsection{Methods}

\subsubsection{Processing}

Two of the foetuses were dissected immediately after hysterotomy and the ovaries fixed in a $4 \%$ phosphate buffered $(0.06 \mathrm{M}, \mathrm{pH} 7.2)$ glutaraldehyde solution. The ovaries of foetus $B$ were prefixed by injection of $2,5 \%$ glutaraldehyde through the umbilical cord prior to dissection. The latter method gave the best fixa- 
tion of the tissue. Thereafter the ovaries were cut in approx. $0.2 \mathrm{~mm}^{3}$ pieces and placed in fresh glutaraldehyde solution for 3 hours at room temperature. After two $15 \mathrm{~min}$ washes in phosphate buffer the tissue was transferred to freshly made $2 \% \mathrm{OsO}_{4}$ in $0.06 \mathrm{~m}$-phosphate buffer ( $\mathrm{pH} 7.2$ ) and postfixed for two hours at room temperature while rotated in a carousel. Following two rinses in distilled water, the tissue was placed in $2 \%$ aqueous uranyl acetate solution for three hours at $60{ }^{\circ} \mathrm{C}$. Dehydration in a graded alcohol series included the following steps: $25 \%$ ethanol for 15 $\mathrm{min}, 50 \%$ ethanol for $15 \mathrm{~min}, 75 \%$ ethanol for 15 $\min , 90 \%$ ethanol for $20 \mathrm{~min}, 96 \%$ ethanol for 20 $\mathrm{min}$ and three times absolute ethanol for $20 \mathrm{~min}$. The tissue was then placed in propylene oxide for $20 \mathrm{~min}$, passed through a graded propylene oxide - Spurr resin series, embedded in pure resin and polymerized overnight at $70^{\circ} \mathrm{C}$.

Survey sections of $2 \mu \mathrm{m}$ thickness were analyzed under the light microscope and groups of cells in the desired meiotic stage were selected for serial sectioning. Thin sections of approximately $0.08 \mu \mathrm{m}$ thickness (pale gold interference colour) were cut in series of 200-300 with a diamond knife on a Reichert Om U3 ultramicrotome, collected on an uncoated single slot grid and transferred with the aid of a micromanipulator to a formvar covered slot grid. The sections were stained in $5 \%$ aqueous uranyl acetate for $30 \mathrm{~min}$ at $60^{\circ} \mathrm{C}$ and in lead citrate for $30 \mathrm{~min}$ at $20^{\circ} \mathrm{C}$ in an LKB Ultrostainer 2168, System Carlsberg.

Grids were examined in a Siemens 102 electron microscope at $80 \mathrm{kV}$. Sections of the selected nuclei were photographed at a magnification of 3000 or 4000 times and printed to a final magnification of $13-20,000$ times.

\subsubsection{Three dimensional reconstruction}

Stretches of the synaptonemal complexes from ten successive micrographs were drawn on transparent plastic sheets. Having encompassed the entire nucleus the tracings of all synaptonemal complex segments of individual bivalents were redrawn on new sheets of plastic film together with the relevant section numbers. The projected lengths were measured and, considering the section thickness, the real lengths calculated using the Pythagorean theorem. The measurements and calculations were carried out on a
Hewlett Packard digitizer and calculator (HP 9864A and 9825A). Finally, the karyotype of each nucleus together with all relevant designations such as centromeres, recombination nodules, structural markers etc. were plotted on an HP 9872 plotter.

\section{RESULTS}

\subsection{Histological organization of the ovary}

As the ovarian cortex develops, it becomes divided by ingrowing connective tissue and blood vessels and appears in cross section as tightly packed clusters of germ cells and granulosa cells surrounded by connective tissue.

In the ovary from the 16-17 week old foetus (A), the clusters were composed mainly of oogonia and oocytes at leptotene and zygotene, although mitotic and pachytene cells were also seen. In the two older ovaries, oocytes at late zygotene and pachytene dominated together with degenerating oocytes and irregular granulosa cells (Figure 1) with dense, elongated nuclei. In the periphery of most clusters, diplotene oocytes surrounded by granulosa cells were also present.

Cells within the same cluster can thus differ considerably with respect to developmental stage as also observed by BAKER (1).

\subsection{Substages of zygotene}

Nuclei described in the following are arranged in a temporal sequence according to the degree of chromosome pairing and synaptonemal complex formation, as other morphological parameters were found to exhibit considerable variation among the nuclei.

\subsubsection{Leptotene-zygotene transition}

The leptotene stage is characterized by formation of the electron dense lateral components of the synaptonemal complex between the two sister chromatids of each chromosome. Organization of lateral components may be initiated interstitially as well as at the telomeres, which in most cases are attached to the inner membrane of the nuclear envelope. In cross section, the lateral components are round or ellipsoid and approximately $40 \mathrm{~nm}$ in diameter.

In the three partially reconstructed nuclei allo- 


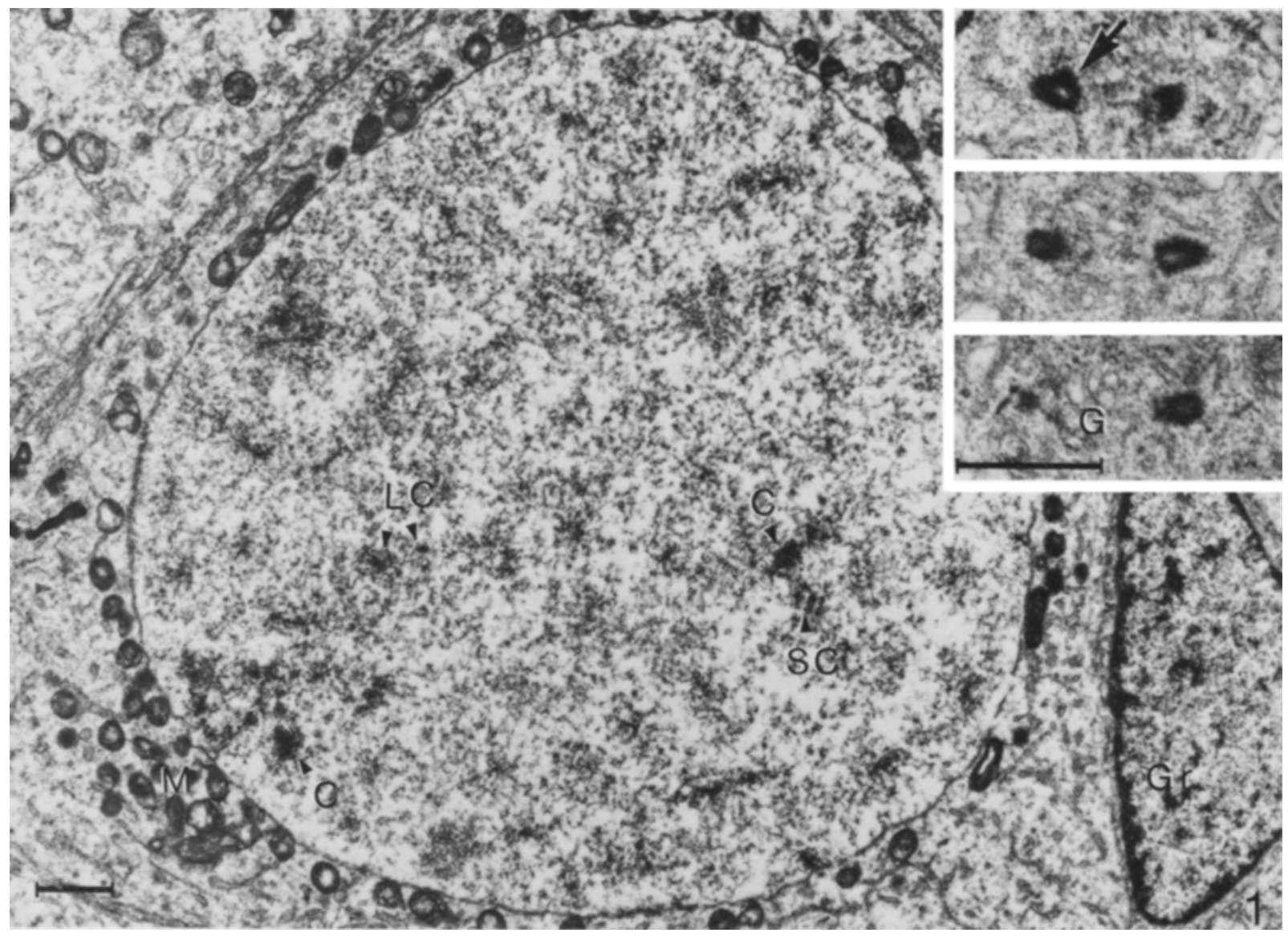

Figure 1. Survey micrograph of a nucleus at the leptotene-zygotene transition. Several unpaired lateral components (LC) and one synaptonemal complex (SC) are seen in cross section. Two centromere regions (C) are distinguished by their condensed chromatin. A layer of mitochondria $(\mathrm{M})$ surrounds the nucleus. Note the granulosa cell $(\mathrm{Gr})$ with its nucleus to the right. $(\mathrm{Bar}=1 \mu \mathrm{m})$.

Insert: Three consecutive sections through a pair of centrioles within an aggregate of Golgi vesicles $(G)$. Note a procentriole (arrow). (Bar $=1 \mu \mathrm{m})$.

cated to this stage, 68 (Figures 1 and 2), 86 and 92 telomeres were associated with the nuclear envelope. The latter nucleus, in which all the telomeres were attached to the envelope, had almost continuous lateral components and was thus the most advanced. The attachment sites of the telomeres in the two early leptotene nuclei were distributed more or less evenly throughout the nuclear envelope (Figure 2), while those of the late leptotene nucleus had aggregated within a limited area, giving rise to a distinct chromosome bouquet.

The pairing of homologous chromosomes and synaptonemal complex formation were initiated in all three nuclei, as evidenced by short (0.2$0.5 \mu \mathrm{m}$ ) stretches of synaptonemal complex. Since the lateral components were as yet incomplete, it indicates that a leptotene stage with fully organized lateral components but without synaptonemal complexes may not exist in human oocytes.

The synaptonemal complex consists of two lateral components combined by a $110 \mathrm{~nm}$ wide amorphous central region with a $20 \mathrm{~nm}$ central component of medium density. The centromere regions can be identified as relatively more condensed chromatin regions (Figure 1), while the remainder of the chromatin appears diffuse, the 


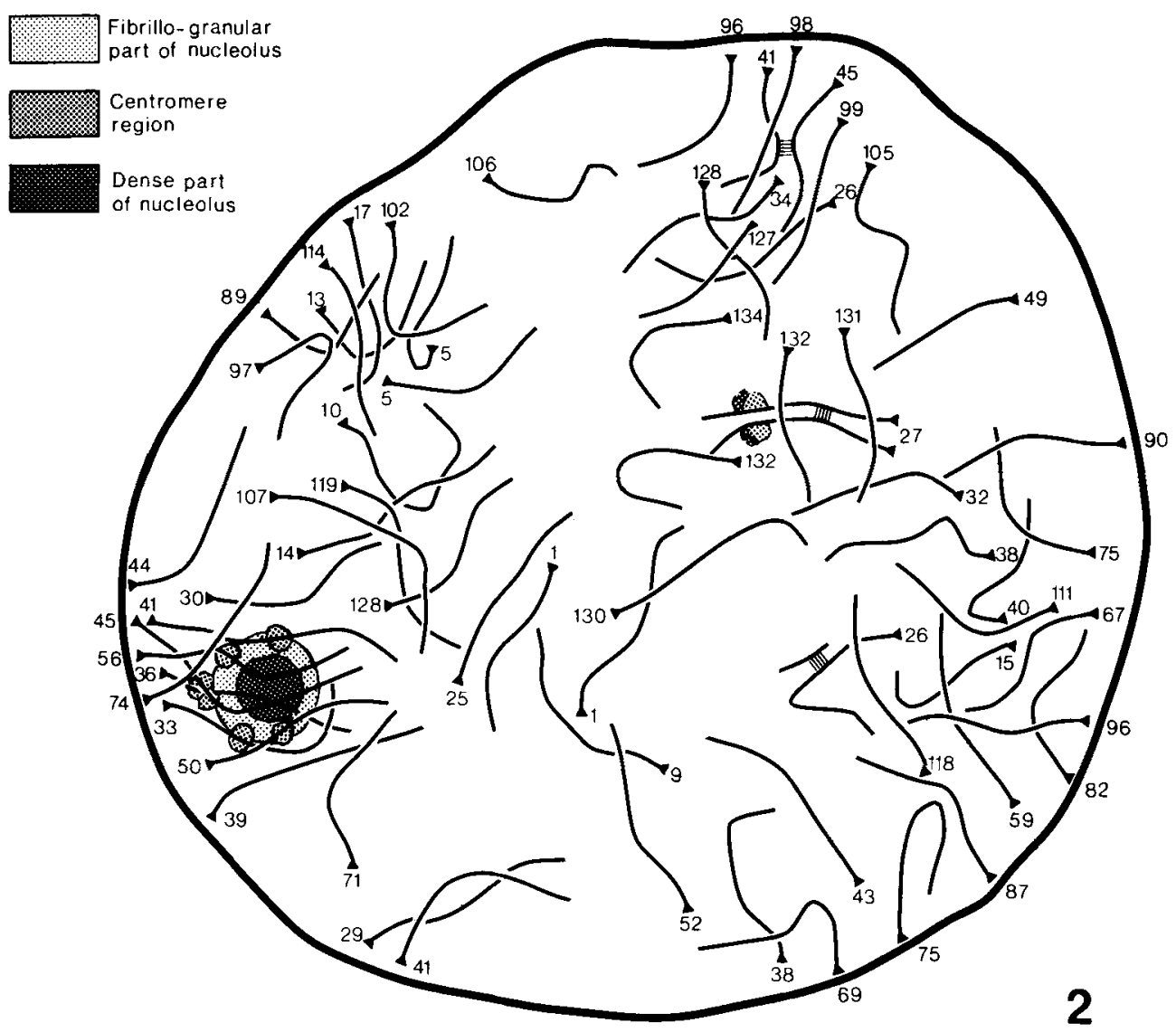

Figure 2. Partial reconstruction of a nucleus at the leptotene-zygotene transition. Sixty-eight of the 92 telomeres are attached to the nuclear envelope, most of the attachment sites being grouped in four areas. Chromosome pairing with synaptonemal complex formation is initiated in three chromosome pairs. Two of the ten acrocentric chromosomes are associated with a small nucleolus, six are associated with the same large nucleolus, while the remaining two appear to be devoid of a nucleolus and cannot be identified. The numbers refer to the section numbers.

most advanced of the three nuclei exhibiting the highest degree of condensation.

Some of the acrocentric chromosomes can be recognized by their association with nucleoli in all three nuclei (Table I). As illustrated in Figure 3 the nucleoli display considerable morphological diversity. One type is highly irregular (Figure 3a) consisting of a dense reticulum associated with the nucleolus organizing region by chromatin-like material. It is likely that the small, globular micronucleoli also present in the nucleus originate from this type of nucleolus. The second type (Figure $3 b$ ) consists of an irregular, fibrillar part which has a density similar to that of the chromatin, containing minor, dense bodies.
The third type (Figure 3c) is spherical and consists of an electron dense spherical core surrounded by fibrillo-granular material of lower electron density.

\subsubsection{Zygotene}

In the five fully reconstructed nuclei, the telomeres were all attached to the nuclear envelope by a conical thickening. The distribution of the attachment sites was distinctly polarized, resulting in a prominent bouquet (Figures 4,5 and 6). Chromosome pairing and synaptonemal complex formation (see section 3.2.1.) was in progress and the lateral components were in all nuclei continuous from telomere to telomere. The 
Table I.

Nucleolus organizing chromosomes and the number of attached and free nucleoli in eight oocytes at the leptotenezygotene transition, mid zygotene and late zygotene.

\begin{tabular}{|c|c|c|c|c|c|c|c|c|}
\hline \multirow[b]{2}{*}{ Stage } & \multicolumn{5}{|c|}{ Nucleolus organizing chromosomes } & \multicolumn{3}{|c|}{ Number of nucleoli } \\
\hline & 13 & 14 & 15 & 21 & 22 & a & $\mathbf{b}$ & Total \\
\hline Leptotene- & \multicolumn{5}{|c|}{8 chromosomes } & 3 & 1 & 4 \\
\hline zygotene & \multicolumn{5}{|c|}{8 chromosomes } & 2 & 1 & 3 \\
\hline transition & \multicolumn{5}{|c|}{6 chromosomes } & 6 & 0 & 6 \\
\hline Mid & - & + & + & + & + & 4 & 2 & 6 \\
\hline zygotene & + & + & + & + & + & 5 & 0 & 5 \\
\hline Late & + & + & + & + & + & 3 & 4 & 7 \\
\hline \multirow[t]{2}{*}{ zygotene } & + & - & - & - & + & 2 & 1 & 3 \\
\hline & + & + & - & - & + & 1 & 2 & 3 \\
\hline
\end{tabular}

a) nucleoli associated with acrocentric chromosomes

b) free nucleoli
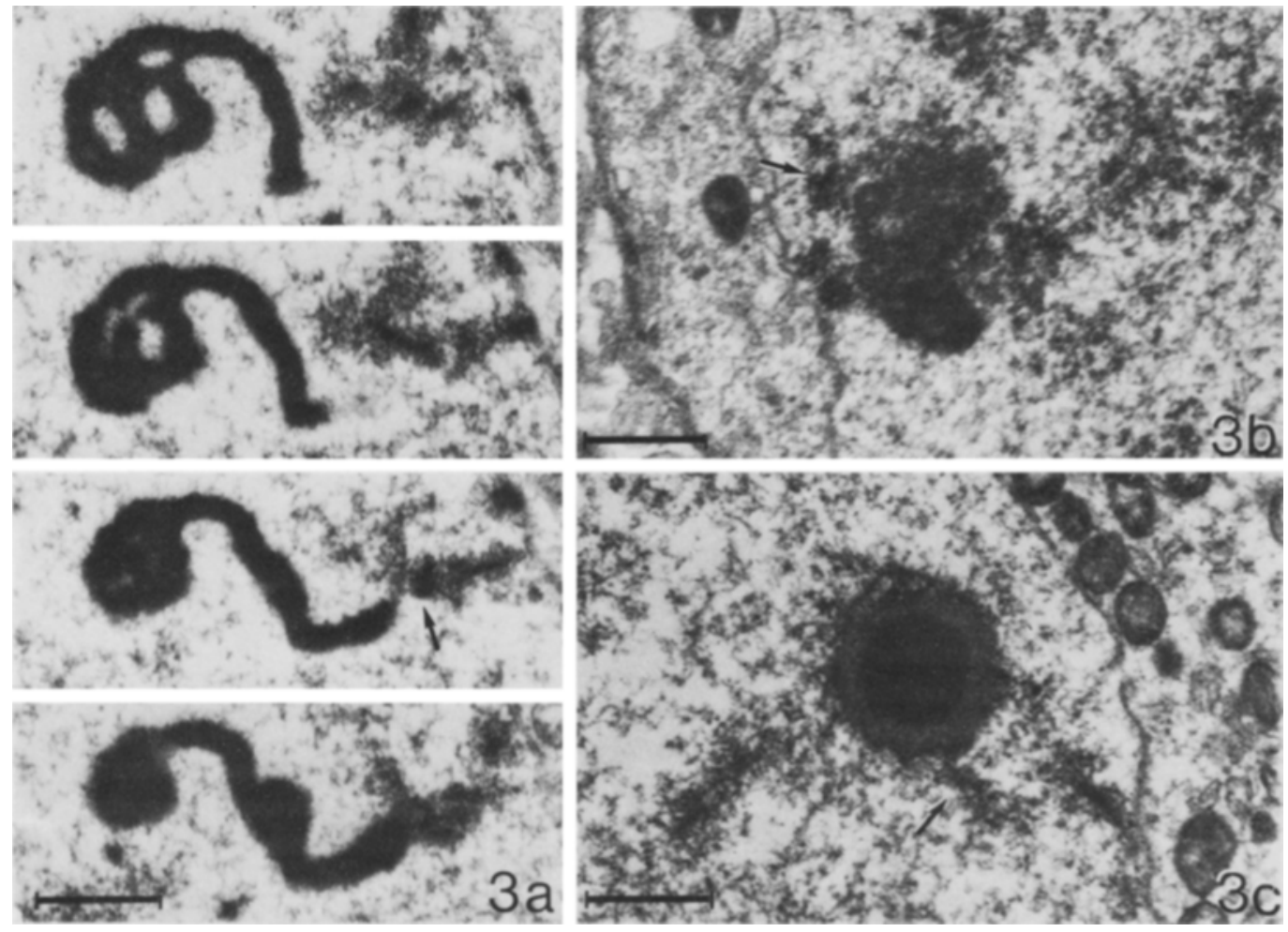

Figure 3. Major nucleoli of different morphology from three oocytes at the leptotene-zygotene transition. Arrows denote the lateral components of acrocentric chromosomes. (Bar $=1 \mu \mathrm{m}$ ). 


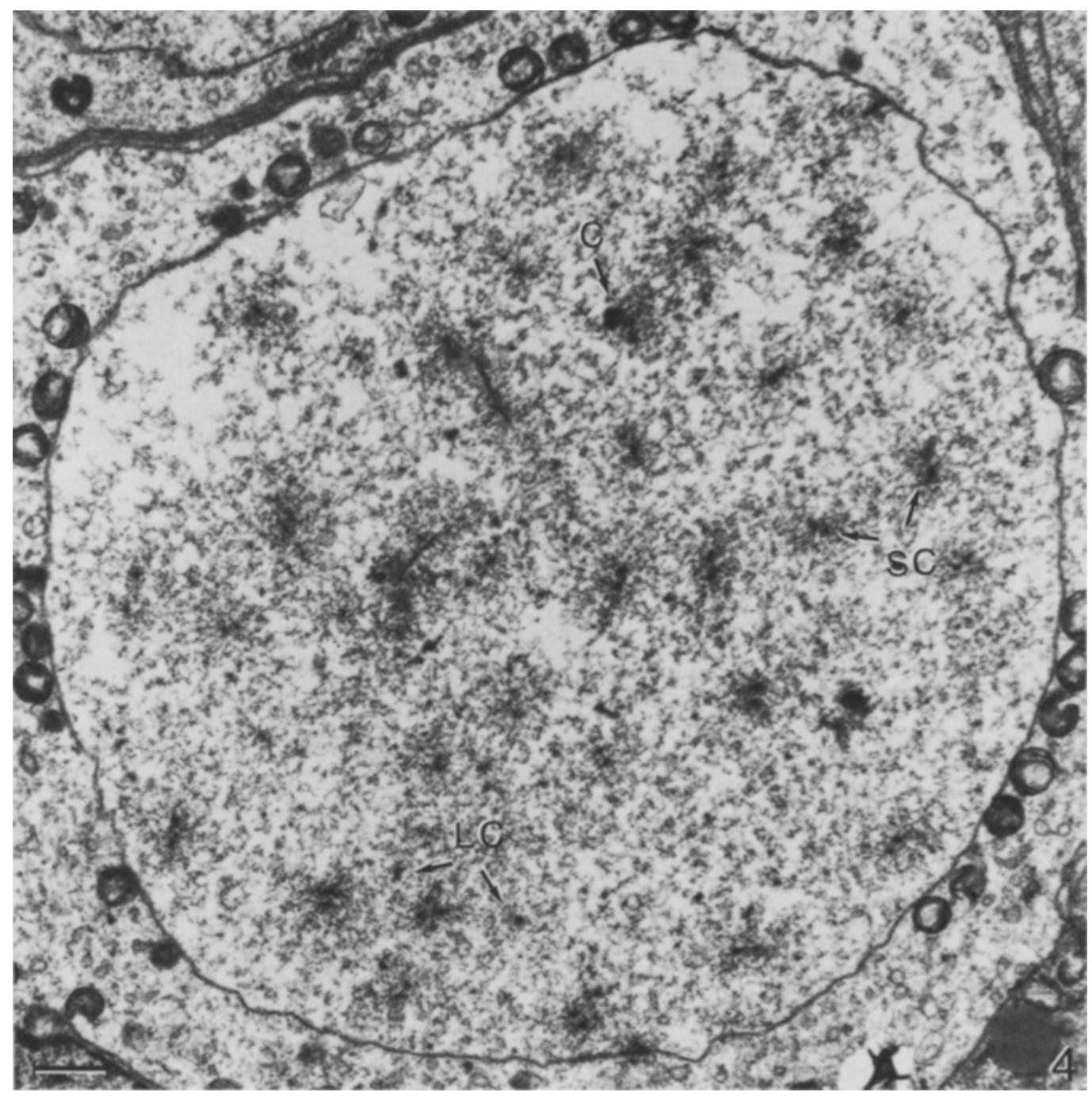

Figure 4. Survey micrograph of a mid zygotene nucleus. Synaptonemal complexes (SC) and unpaired lateral components (LC) are seen in cross and obligue section, the plane of the section being at right angles to the bouquet. Note the decondensed chromatin of the chromosomes and the highly condensed centromeric chromatin (C). (Bar $=1 \mu \mathrm{m}$ ).

five nuclei could be allocated to two substages, two of the nuclei being at mid zygotene with 55 and 67 percent of the complement paired while the remaining three nuclei were at late zygotene with synaptonemal complex formation being almost completed $(97,98$ and 99 percent of the complement paired with a synaptonemal complex (Table II)).

The chromatin becomes more condensed as the pairing proceeds. The chromatin of the mid zygotene nuclei is dispersed (Figure 4), while that of the more advanced oocytes is condensed along the synaptonemal complexes, giving the bivalents a distinct outline (Figure 6). The centromere regions are readily distinguished at mid zygotene as spherical, highly condensed structures with a diameter of approximately $0.4 \mu \mathrm{m}$ (Figure 4), while at later stages the decondensation of the centromeric chromatin makes an unequivocal identification more difficult. 


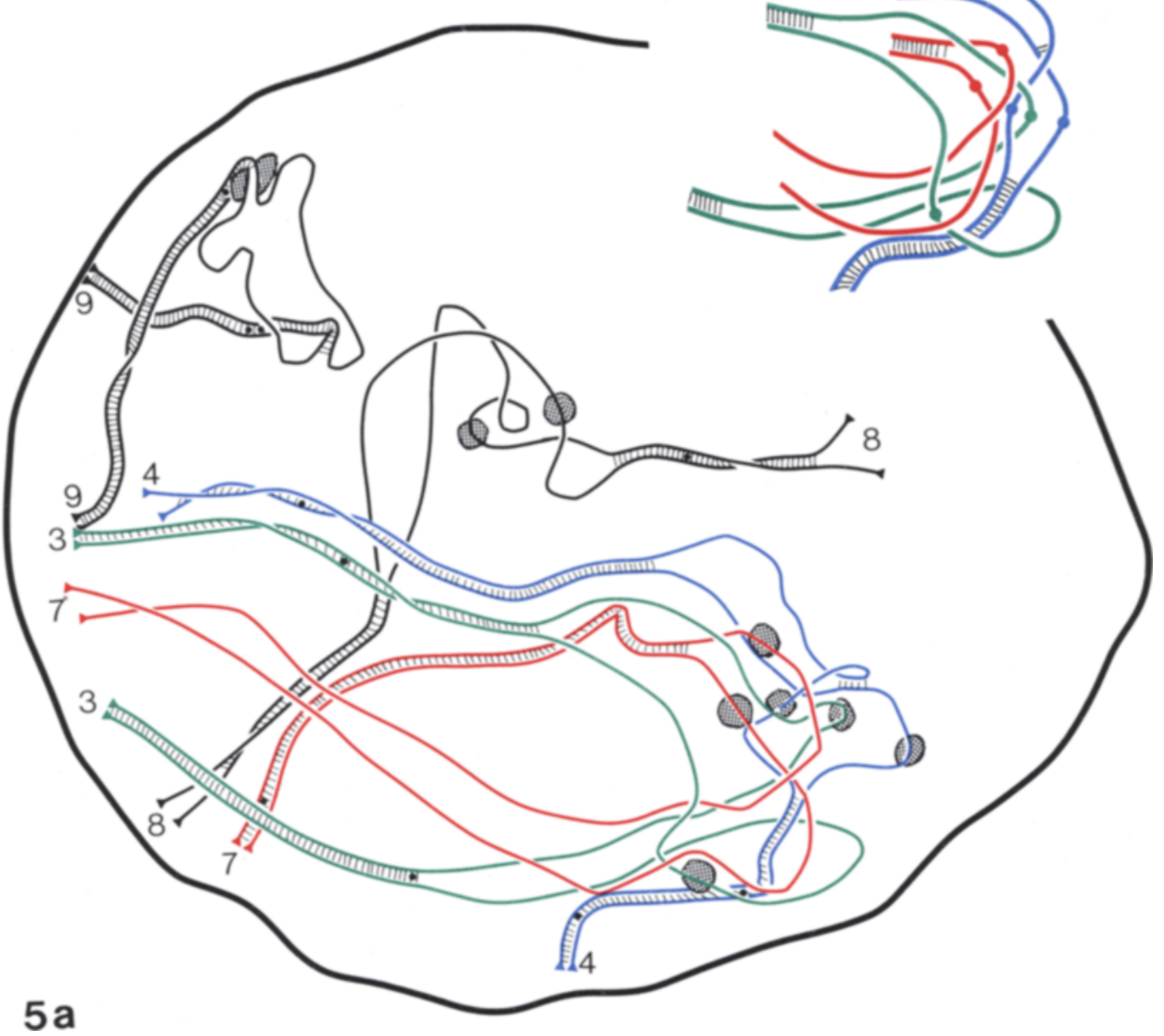

Figure 5. A complete reconstruction of a mid zygotene nucleus (Nucleus A in Table V) displays the chromosome bouquet with all telomeres attached to a limited region of the nuclear envelope. The formation of the synaptonemal complex is completed in bivalent 21 , while the remaining bivalents are only partially paired. Some of these are involved in interlockings ( $5 \mathrm{a}$ and 5c). A schematic drawing of the entangled bivalents 3 (green), 4 (blue) and 7 (red) is shown in the insert (see also Table $\mathrm{V}$ and text). A chromosome break (asterisk) is present in one chromosome 2 (5c). A major nucleolus is attached to the centromeric chromatin of bivalent 21 and four minor nucleoli are associated with bivalents 15, 22 and the unpaired arms of bivalent 14 (see also Figure 9). Recombination nodules are denoted by filled circles and nodule-bar intermediatees by dashes. The centromere regions and nucleoli are hatched as in Figure 2. 


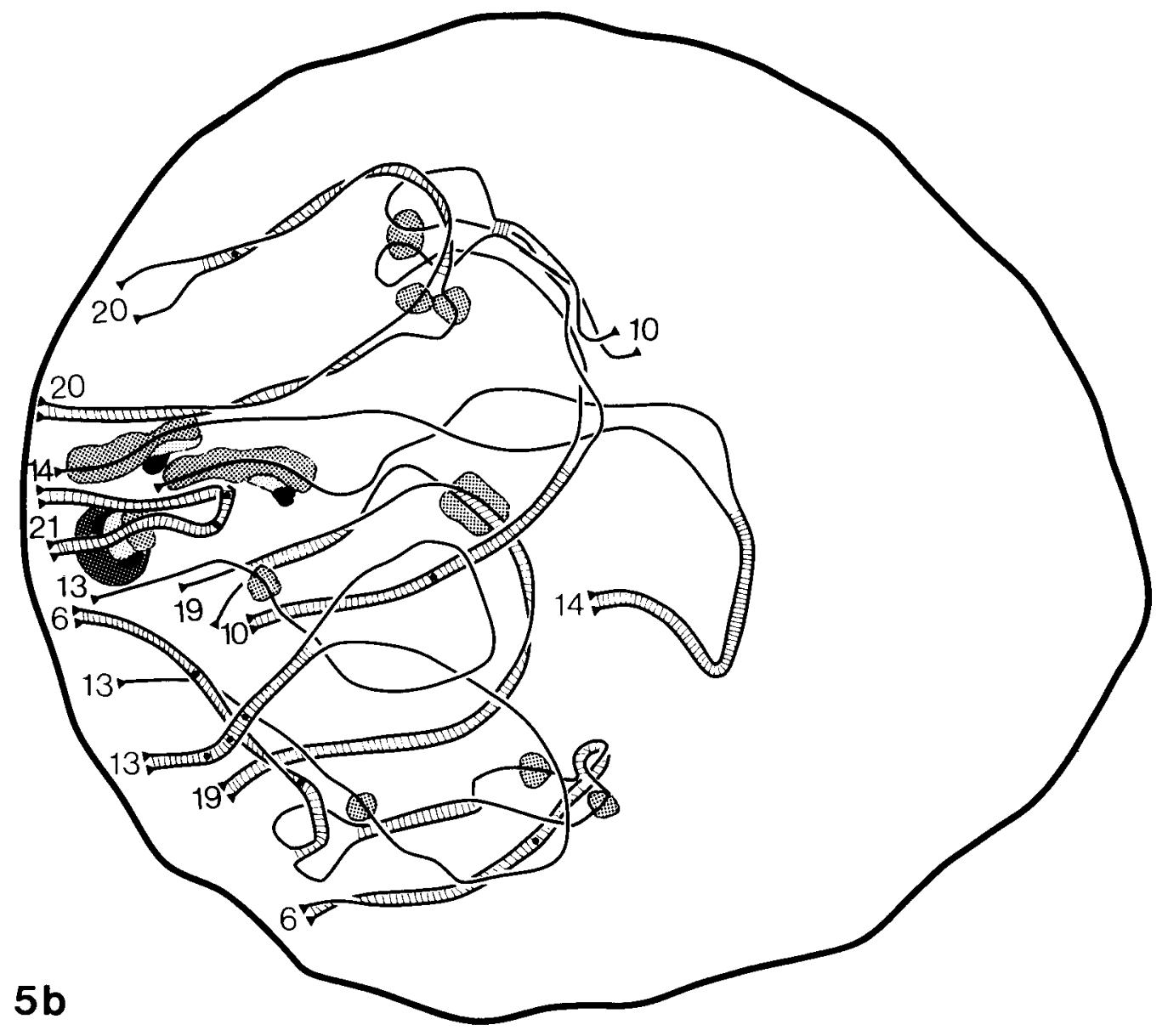


M. BoJKo: Meiosis in the human oocytes

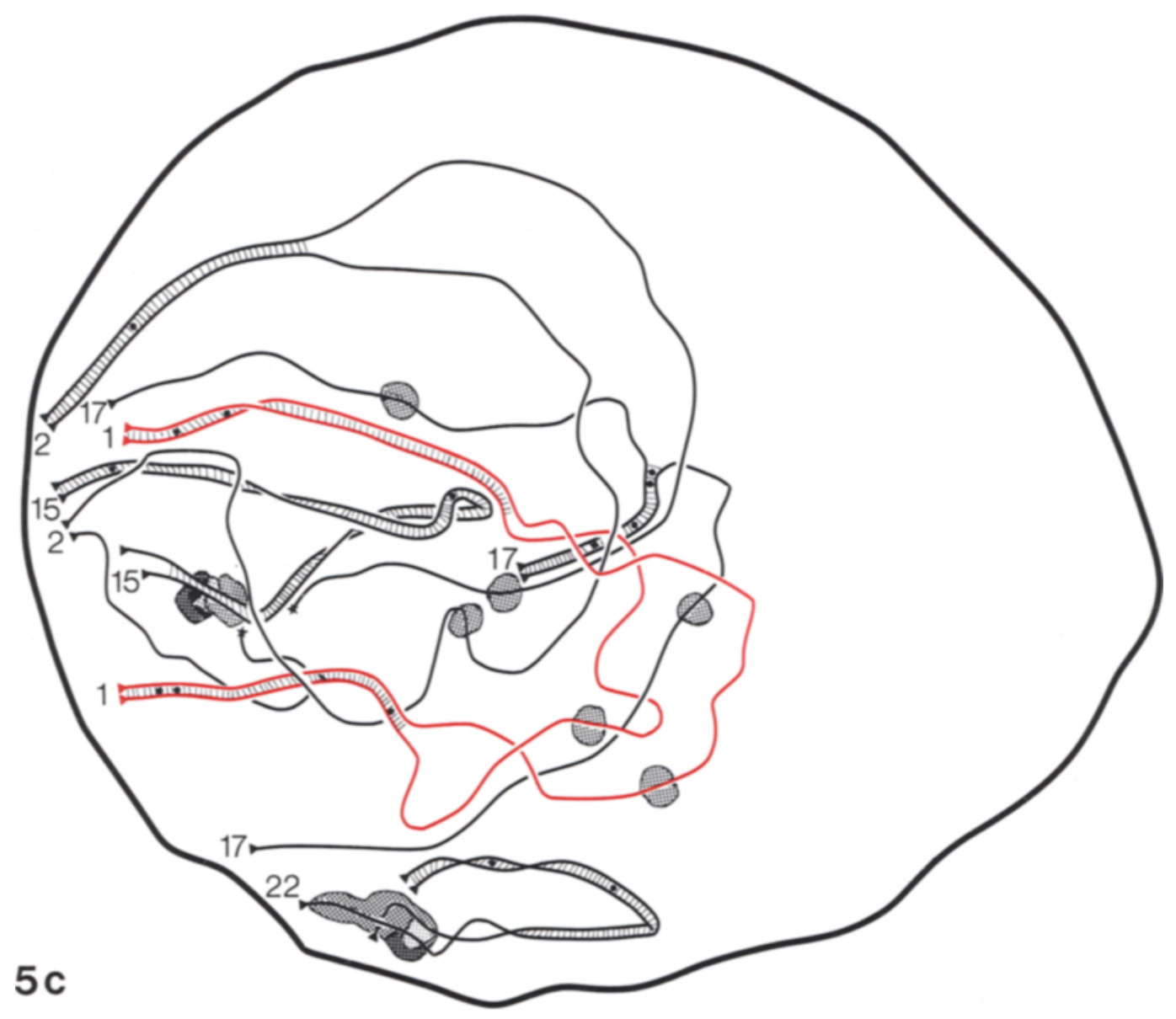


M. Bолко: Meiosis in the human oocytes

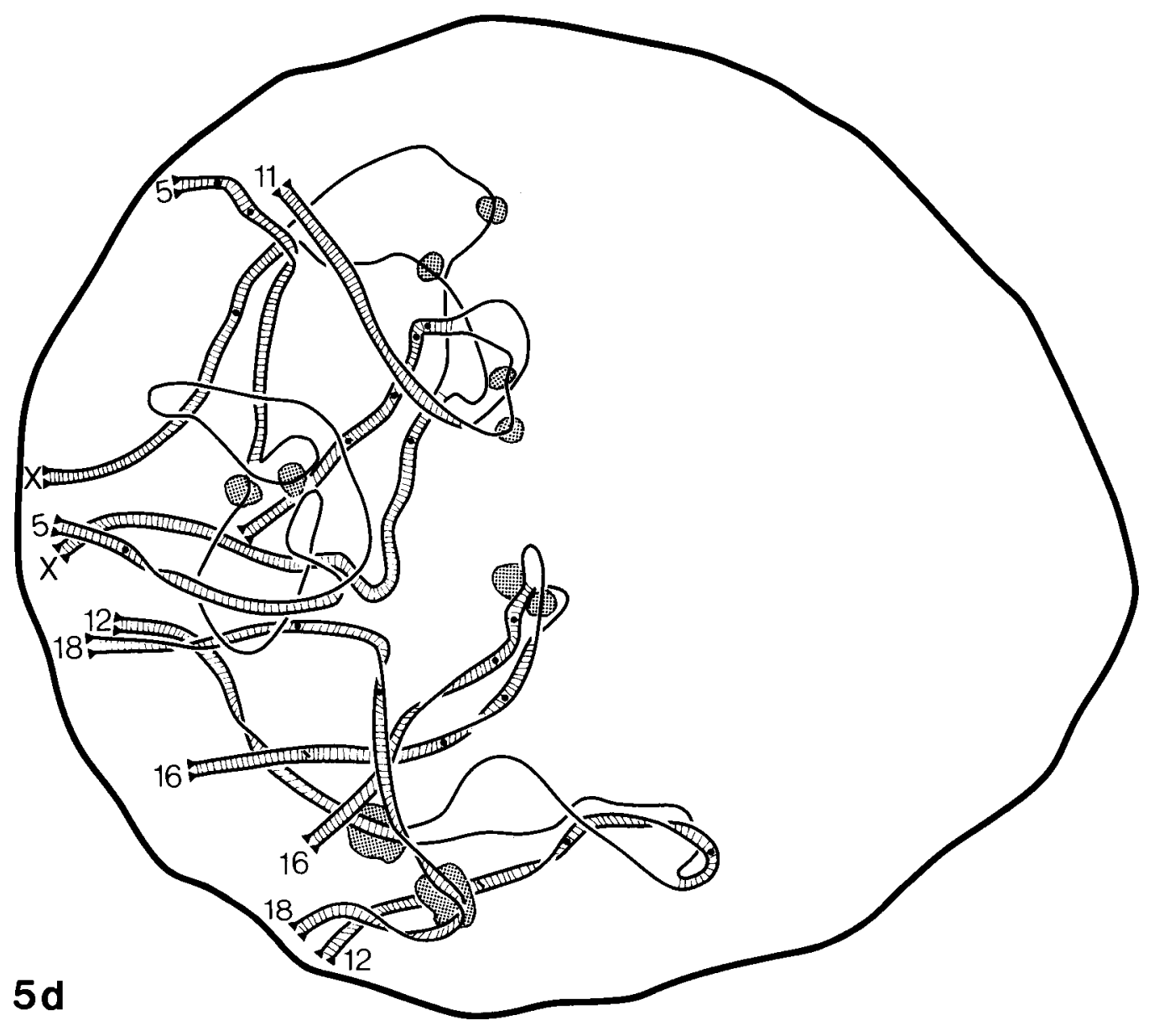




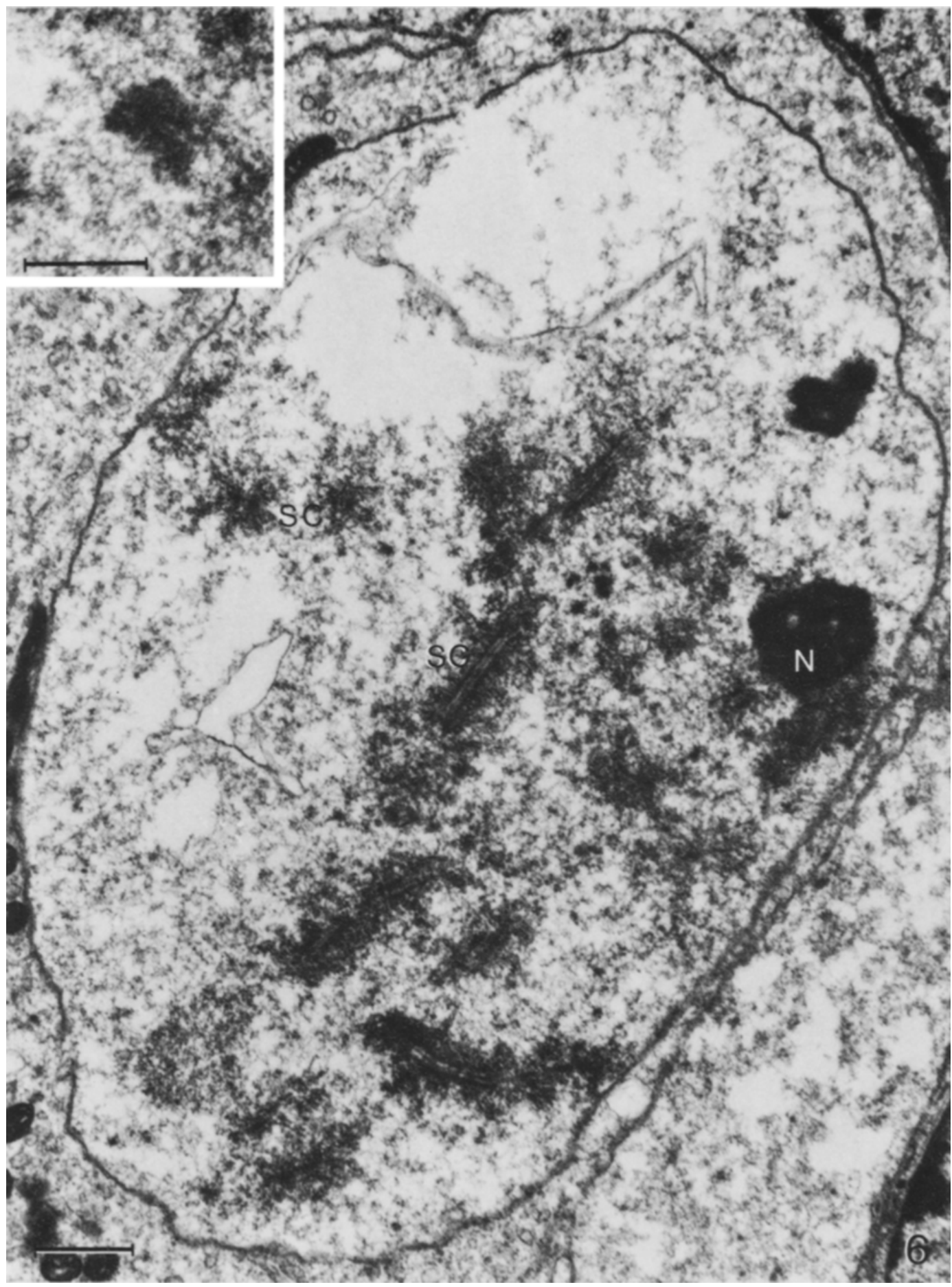

Figure 6. Survey micrograph of a late zygotene nucleus. The bivalents are confined to about one half of the nucleus and the chromatin is more condensed around the synaptonemal complexes (SC) than at mid zygotene (Figure 4). The major nucleolus $(\mathrm{N})$ is associated with an acrocentric bivalent while a minor one is free in the nucleoplasm. $(\mathrm{Bar}=1 \mu \mathrm{m})$.

Insert. Centromeric chromatin of a late zygotene bivalent. $(\mathrm{Bar}=1 \mu \mathrm{m})$. 
Table II.

Total lateral component (LC) length of one genome, total length of synaptonemal complexes (SC), percent pairing, number of recombination nodules (RN) and number of nodule-bar intermediates (RB) for two mid and three late zygotene nuclei.

\begin{tabular}{lcccccc}
\hline Stage & $\begin{array}{c}\text { Total LC } \\
\text { length of one } \\
\text { genome }(\mu \mathrm{m})\end{array}$ & $\begin{array}{c}\text { Total SC } \\
\text { length } \\
(\mu \mathrm{m})\end{array}$ & $\begin{array}{c}\text { Percent } \\
\text { pairing }\end{array}$ & $\begin{array}{c}\text { Number } \\
\text { of } \\
\text { RN }\end{array}$ & $\begin{array}{c}\text { Number } \\
\text { of } \\
\text { RB }\end{array}$ & $\begin{array}{c}\text { Total } \\
\text { number of } \\
\text { RN and RB }\end{array}$ \\
\hline Mid & 413 & 228 & 55 & 55 & 5 & 60 \\
zygotene & 517 & 347 & 67 & 40 & $15 \mathrm{a})$ & 55 \\
\hline Late & 509 & 492 & 97 & 71 & 7 & 78 \\
zygotene & 518 & 510 & 98 & 58 & 4 & 62 \\
& 409 & 406 & 99 & 67 & 2 & 69 \\
\hline
\end{tabular}

a) Seven of these recombination structures were well defined bars, see sect. 3.5 .
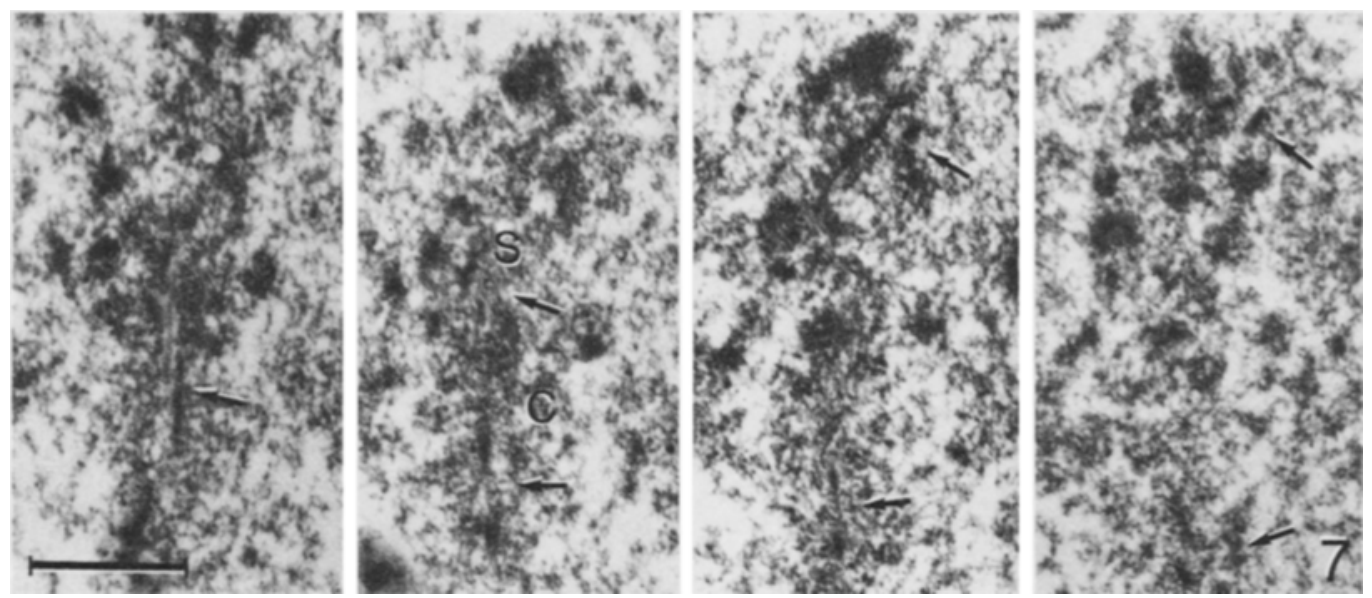

Figure 7. Four consecutive sections through the secondary constriction of bivalent 1 in a late zygotene nucleus. The centromeric chromatin (C) is confluent with the chromatin (S) of the secondary constriction and of the same electron density. Arrows denote the synaptonemal complex. $(\mathrm{Bar}=1 \mu \mathrm{m})$.
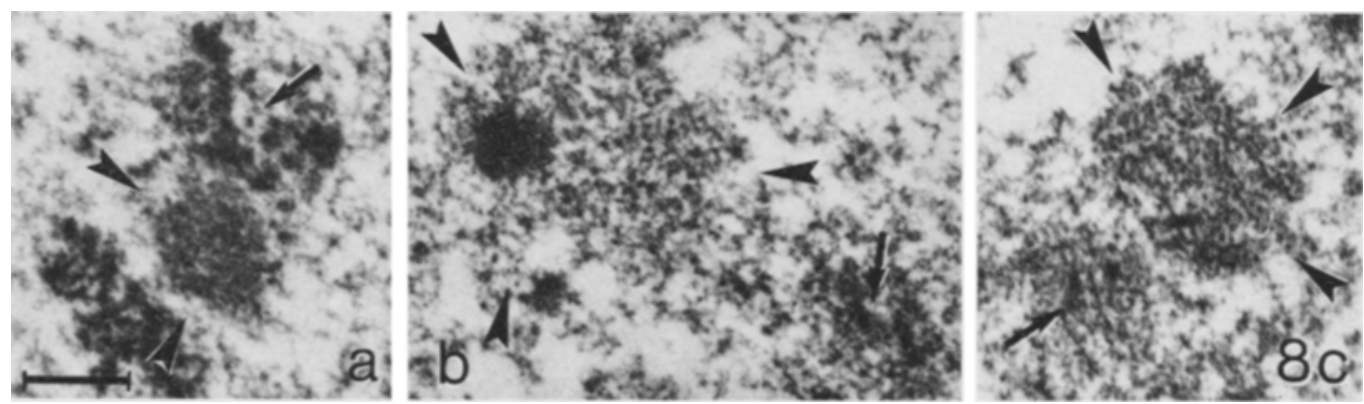

Figure 8. Structural markers in the form of granular-fibrillar bodies on late zygotene chromosomes 6 (a), l (b) and 5(c). The synaptonemal complexes of the bivalents are denoted by arrows and the markers by arrowheads. (Bar $=$ $0.5 \mu \mathrm{m})$. 

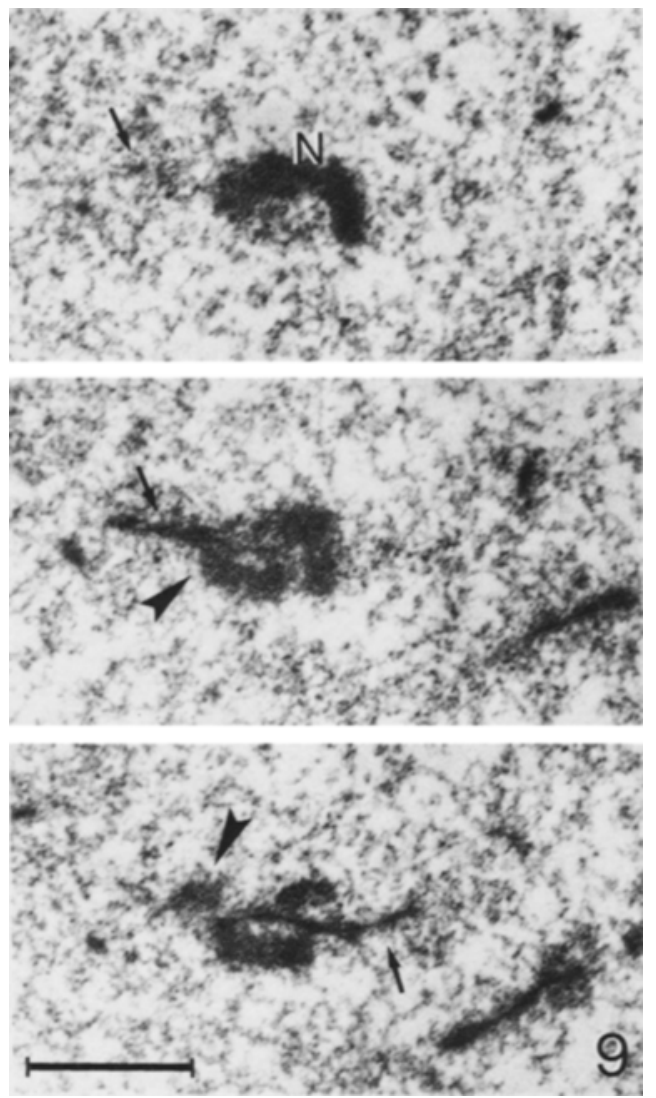

Figure 9. Three consecutive sections showing a nucleolus $(\mathrm{N})$ on an unpaired arm of a mid zygotene chromosome. Arrowheads denote centromeric heterochromatin, arrows denote the lateral component. $(\operatorname{Bar}=1 \mu \mathrm{m})$.

The secondary constriction on bivalents 1,9 and 16 can, as in spermatocytes (28), be identified at all substages of zygotene as a large, spherical or elongated region of diffuse chromatin with numerous, compact knots (Figure 7).

The structural markers previously reported from pachytene spermatocytes (3) on bivalents 1 and 6 were also present in the oocytes. Marker 1 (Figure 8b) consists of a dense part surrounded by irregularly shaped granular-fibrilar material and is located approximately $1.4 \mu \mathrm{m}$ from the telomere of the short arm of bivalent 1. Marker 6 (Figure 8a), located near the middle of the short arm of bivalent 6 is a spherical body composed of fine granules and fibers. Both markers appear during late zygotene and are not seen in unpaired chromosomes.

Besides these well characterized markers, a variety of other structures were observed, some of which may prove to be useful in future chromo-
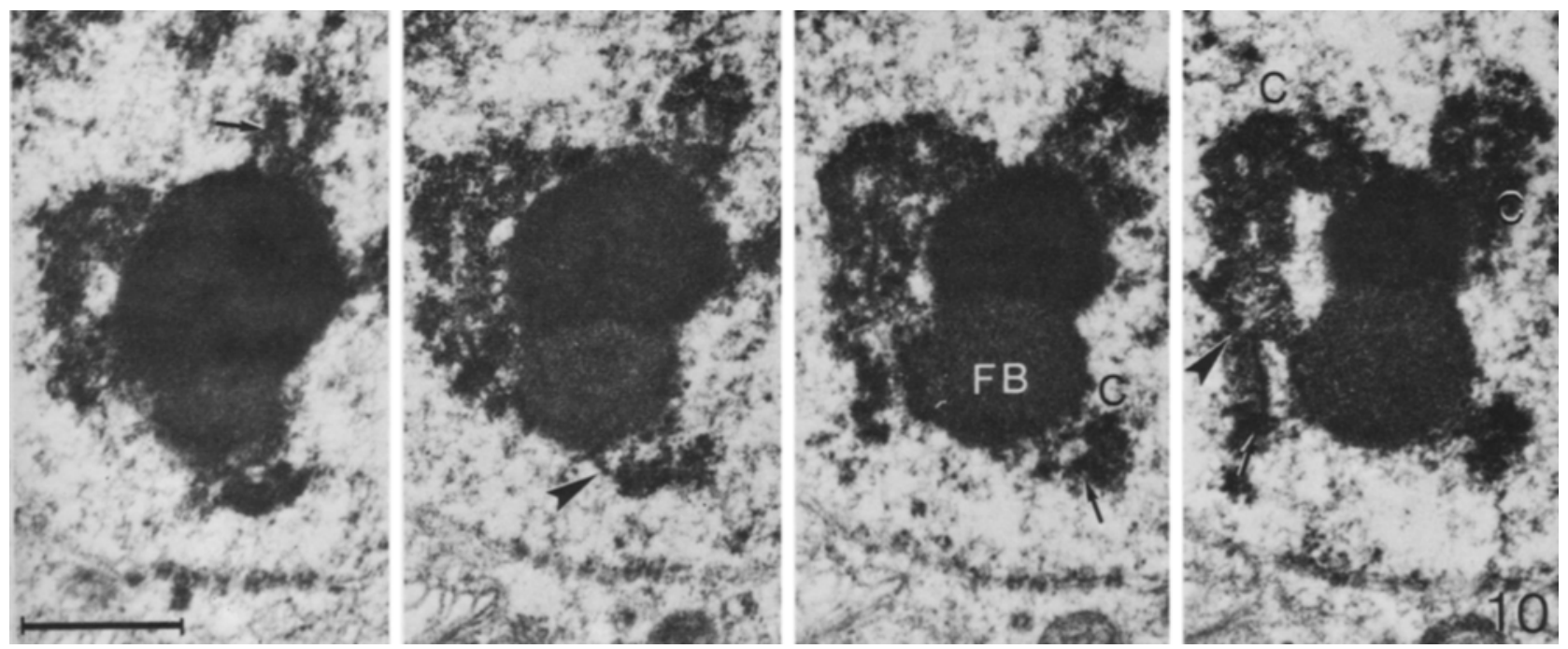

Figure 10. Four consecutive sections through a major late zygotene nucleolus associated with three acrocentric bivalents (arrows). The fibrillar body (FB) of the nucleolus is connected to two of the bivalents through chromatinlike material (arrowheads) near the centromeric chromatin $(C) .(B a r=1 \mu \mathrm{m})$. 
Table III.

Mean absolute and relative lengths and centromere indices of the 23 bivalents from 5 zygotene oocytes and 10 zygotene spermatocytes. The relative lengths of the bivalents from the spermatocytes are based on the total autosomal length, while in the oocytes the relative lengths are calculated from the combined length of the autosomes and the $\mathrm{X}$ bivalent (sd, standard deviation).

\begin{tabular}{|c|c|c|c|c|c|c|c|c|c|c|c|c|}
\hline \multirow{3}{*}{$\begin{array}{l}\text { Bivalent } \\
\text { number }\end{array}$} & \multicolumn{4}{|c|}{ Absolute length } & \multicolumn{4}{|c|}{ Relative length } & \multicolumn{4}{|c|}{ Centromere index } \\
\hline & \multicolumn{2}{|c|}{ Oocytes } & \multicolumn{2}{|c|}{ Spermatocytesa) } & \multicolumn{2}{|c|}{ Oocytes } & \multicolumn{2}{|c|}{ Spermatocytesa) } & \multicolumn{2}{|c|}{ Oocytes } & \multicolumn{2}{|c|}{ Spermatocytesa) } \\
\hline & Mean & sd & Mean & sd & Mean & sd & Mean & sd & Mean & sd & Mean & sd \\
\hline 1 & 39.4 & 9.1 & 19.2 & 2.1 & 8.3 & 1.2 & 8.2 & 2.5 & 44 & 3 & 51 & 5 \\
\hline 2 & 34.0 & 4.8 & 17.4 & 1.9 & 7.2 & 0.6 & 7.5 & 0.5 & 41 & 3 & 40 & 4 \\
\hline 3 & 30.9 & 6.8 & 15.4 & 2.1 & 6.5 & 0.9 & 6.6 & 0.6 & 48 & 2 & 45 & 4 \\
\hline 4 & 25.9 & 2.4 & 14.1 & 1.4 & 5.5 & 0.4 & 6.0 & 2.3 & 33 & 5 & 36 & 5 \\
\hline 5 & 25.0 & 5.2 & 13.7 & 1.6 & 5.7 & 0.4 & 5.8 & 0.1 & 28 & 5 & 30 & 4 \\
\hline 6 & 24.7 & 4.0 & 13.2 & 1.5 & 5.2 & 0.4 & 5.5 & 0.4 & 35 & 3 & 31 & 7 \\
\hline 7 & 24.4 & 2.6 & 12.6 & 1.4 & 5.2 & 0.4 & 5.4 & 0.2 & 40 & 2 & 37 & 7 \\
\hline 8 & 20.7 & 2.6 & 11.1 & 1.3 & 4.4 & 0.2 & 4.7 & 1.9 & 36 & 2 & 36 & 4 \\
\hline 9 & 22.2 & 2.4 & 11.8 & 1.0 & 4.6 & 0.2 & 5.1 & 0.3 & 37 & 5 & 37 & 3 \\
\hline 10 & 21.2 & 2.8 & 11.8 & 1.3 & 4.5 & 0.1 & 5.0 & 0.3 & 34 & 3 & 34 & 5 \\
\hline 11 & 18.3 & 2.9 & 10.5 & 1.7 & 3.9 & 0.5 & 4.5 & 0.4 & 37 & 3 & 38 & 6 \\
\hline 12 & 22.2 & 3.1 & 11.4 & 1.4 & 4.7 & 0.2 & 4.9 & 0.3 & 29 & 1 & 28 & 3 \\
\hline 13 & 18.8 & 2.4 & 9.9 & 2.1 & 4.0 & 0.4 & 4.2 & 0.6 & 13 & 5 & 14 & 4 \\
\hline 14 & 17.3 & 1.9 & 8.4 & 1.2 & 3.6 & 0.2 & 3.6 & 0.3 & 11 & 3 & 14 & 5 \\
\hline 15 & 16.2 & 1.9 & 8.0 & 1.3 & 3.4 & 0.1 & 3.4 & 0.2 & 12 & 3 & 15 & 3 \\
\hline 16 & 15.3 & 1.4 & 8.4 & 1.0 & 3.3 & 0.4 & 3.6 & 0.3 & 46 & 2 & 43 & 3 \\
\hline 17 & 16.5 & 2.3 & 8.5 & 1.1 & 3.5 & 0.3 & 3.6 & 0.3 & 32 & 1 & 33 & 3 \\
\hline 18 & 12.4 & 1.3 & 6.3 & 0.7 & 2.6 & 0.3 & 2.6 & 0.2 & 27 & 2 & 26 & 3 \\
\hline 19 & 12.1 & 2.0 & 6.2 & 0.7 & 2.6 & 0.2 & 2.7 & 0.3 & 43 & 4 & 43 & 4 \\
\hline 20 & 13.8 & 1.9 & 8.0 & 1.1 & 2.9 & 0.3 & 3.4 & 0.3 & 45 & 4 & 43 & 4 \\
\hline 21 & 7.8 & 1.6 & 3.6 & 0.6 & 1.6 & 0.2 & 1.6 & 0.1 & 18 & 6 & 26 & 5 \\
\hline 22 & 9.3 & 0.9 & 5.4 & 1.4 & 1.9 & 0.1 & 2.3 & 0.4 & 14 & 5 & 19 & 4 \\
\hline$X$ & 22.9 & 2.1 & 11.8 & 1.9 & 4.8 & 0.2 & & & 42 & 3 & & \\
\hline
\end{tabular}

a) data from RASMUSSEN and HOLM (28).

some identification. One of the candidates is a spherical structure observed in mid zygotene nuclei, consisting of distinct granules located approximately $2.5 \mu \mathrm{m}$ from the telomere of the short arm of chromosome 5 (Figure 8c).

All acrocentric chromosomes are capable of organizing a nucleolus as can be seen in Table I, but some of the acrocentric chromosomes lack a nucleolus except in one mid and one late zygotene nucleus. In addition, most of the mid and late zygotene nuclei contain one or more free nucleoli. Nucleoli organized by unpaired chromosomes are small, round to ovoid and consist of an electron opaque body connected to the chromosomes by a chromatin-like material (Figure 9). Larger nucleoli are attached to one or more bivalents, one type consising of compact, dark bodies surrounded by a less dense, irregular mass of fibrillar material penetrated by electron transparent channels (Figure 6). The other type (Figure 10) is composed of two spherical parts, an electron dense core containing even darker bodies and being connected to the bivalents through a homogenous, fibrillo-granular body of medium electron density.

Outside the nuclear envelope, mitochondria are abundant surrounding the nucleus. This localization of mitochondria is characteristic of human oocytes and has not been reported for other mammalian meiocytes. Many of Golgi apparatuses are aggregated in the vicinity of the two centrioles. The centrioles are separated by a distance of $1-2 \mu \mathrm{m}$ (Figure 1) and each has a small procentriole at right angle to the longitudinal 
ABSOLUTE LENGTH $(\mu \mathrm{m})$
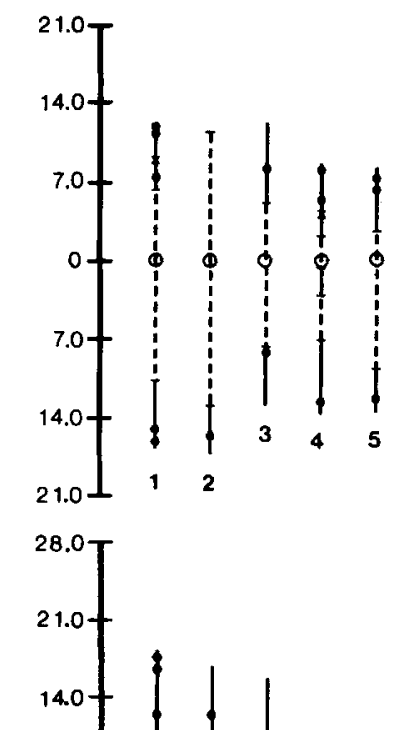

\author{
MID ZYGOTENE
}

LATE ZYGOTENE

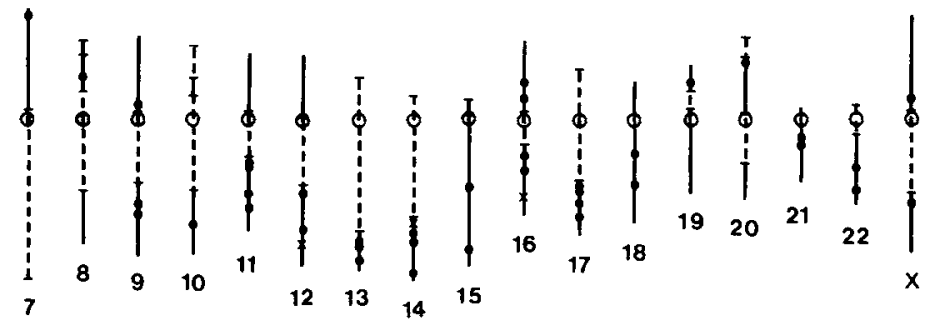
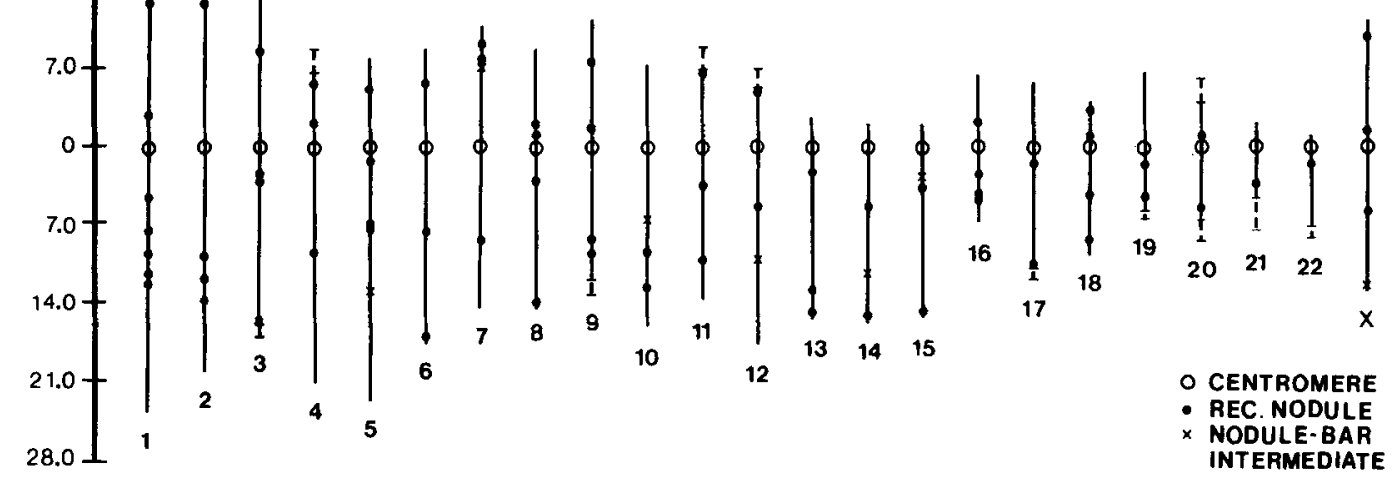

Figure 11. Idiograms of the bivalent complement of a mid and a late zygotene nucleus showing the degree of pairing and the distribution of recombination nodules and nodule-bar intermediates. The regions paired with a synaptonemal complex are shown as continuous lines and the unpaired regions as broken lines.

Table IV.

Total number and location of synaptonemal complex segments in partially synapsed bivalents of two mid (37 bivalents) and three late (16 bivalents) zygotene nuclei.

\begin{tabular}{lcccc}
\hline & $\begin{array}{c}\text { Paired } \\
\text { segments } \\
\text { per bivalent }\end{array}$ & $\begin{array}{c}\text { Number of } \\
\text { partly paired } \\
\text { bivalents }\end{array}$ & $\begin{array}{c}\text { Number } \\
\text { of paired } \\
\text { telomere regions }\end{array}$ & $\begin{array}{c}\text { Number of } \\
\text { interstitially } \\
\text { paired segments }\end{array}$ \\
\hline Mid zygotene & 1 & 11 & 11 & 0 \\
\hline Late zygotene & 2 & 23 & 42 & 4 \\
\hline Total & 3 & 3 & 5 & 4 \\
\hline
\end{tabular}


axis of the mother centriole. No differences are seen in the organization of the cytoplasm and its organelles throughout zygotene.

\subsection{The zygotene karyotype}

The total complement length per nucleus at mid and late zygotene is given in Table III. The total length varies between $409 \mu \mathrm{m}$ and $518 \mu \mathrm{m}$ with a mean value of $473 \pm 57 \mu \mathrm{m}$.

Classification of the individual bivalents was performed according to the criteria decided at the Paris Conference 1971 (24) and to those described for spermatocytes (13), the classification primarily being based on length, centromere index and structural markers.

On the basis of these parameters, chromosomes $1-3,6,9$ and $16-22$ can be identified, while the classification of the remaining chromosomes beyond an assignment to groups $\mathrm{B}, \mathrm{C}$ and $\mathrm{D}$ is more uncertain. The $X$ bivalent appears indistinguishable from the autosomal bivalents at zygotene and thus cannot be identified morphologically. The relative length and centromere index allocate it to group $\mathrm{C}$, and the $\mathrm{X}$ bivalent has tentatively been identified as the one with the highest centromere index among the three longest chromosomes of the group containing bivalents $8,10,11$ and 12 .

Table III presents the mean absolute and relative lengths and centromere indices of the bivalents from five oocytes at mid and late zygotene and ten spermatocytes at late zygotene (28). As can be seen from the table, the karyotypes are very similar with respect to relative lengths and centromere indices, whereas the absolute lengths of the bivalents of the spermatocytes are approximately half as long as those of oocytes. The karyotype of a mid and a late zygotene nucleus is shown in Figure 11.

\subsection{Chromosome pairing}

\subsubsection{Formation of the synaptonemal complex}

The reconstructions in Figure 5 show a nucleus at mid zygotene, illustrating together with the idiograms in Figure 11 typical features of chromosome pairing and synaptonemal complex formation.

The number and location of synaptonemal complex segments in the partially paired bivalents in the two mid zygotene and three late zygotene nuclei (Table IV) show that pairing and synaptonemal complex formation in most cases start at or near the telomeric region, as 76 out of 85 paired segments include a telomere. Twentyfour of the 53 bivalents paired from one, and 26 paired from both telomeres, the remaining three bivalents showing only interstitial pairing. Interstitially paired segments were observed 9 times, in two of the cases an interlocking was present in the vicinity. Centromere regions were paired in 13 of the 37 partially synapsed mid zygotene bivalents and in all late zygotene bivalents. The delayed pairing in the secondary constriction on bivalents 1,9 , and 16 noted at zygotene and early pachytene in human spermatocytes (28) was not observed in the analyzed oocytes.

In several cases, spherical, dense structures 100 to $150 \mathrm{~nm}$ in diameter were present on the cytoplasmic side of the attachment sites of the nuclear envelope, and appeared to be connected to paired as well as unpaired telomeres by a bundle of filaments (Figure 12). The maximum number of these spheres was found at mid zygotene, whereas only few were present at early pachytene. Similar structures are present in spermatocytes during zygotene and early pachytene (HOLM and Rasmussen, personal communication), and mid pachytene (13), and it is conceivable that telomere movements on the nuclear envelope somehow are mediated through these structures. In agreement with this proposal; PARvinen and Söderström (25) reported that rotatory and oscillatory chromosome movements are maximal at early and mid zygotene and gradually cease during early pachytene. So far, similar nodes have not been reported in other organisms and they may accordingly represent a species specific differentiation of the apparatus responsible for aggregation of the telomeres during zygotene.

\subsubsection{Chromosome interlocking and breakage}

Interlockings and breakage of chromosomes and bivalents were frequent in the two mid zygotene nuclei, in the following referred to as nuclei $A$ and $B$ (Figures 5 and 13). As shown in Table $V$ and Figure 5, nucleus $A$ possesses one bivalent interlocking, five chromosome interlockings and one chromosome break. The 


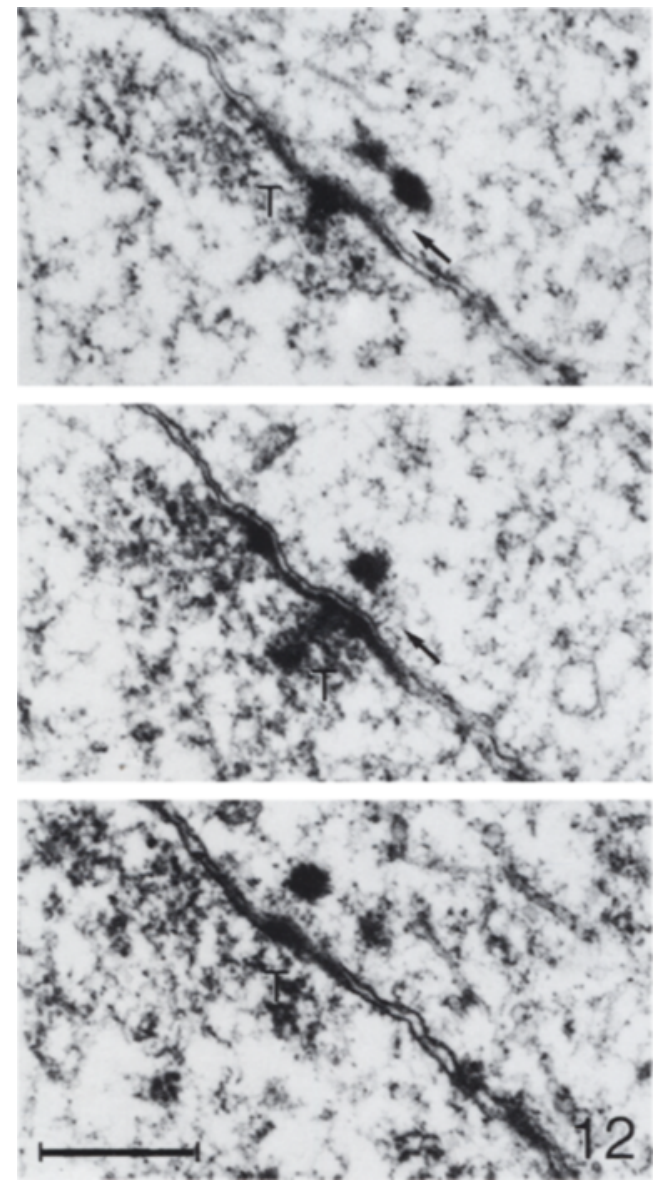

Figure 12. Three attachment sites of lateral components on the nuclear envelope. Note that an electron dense sphere is present on the cytoplasmic side of the attachment sites and that the spheres appear to be connected to the ends of the lateral components by filaments. (Bar $=1 \mu \mathrm{m})$.

telomeres of the interlocking homologues of bivalent 7 were only $0.7 \mu \mathrm{m}$ apart, but no synaptonemal complex was organized distal to the interlocking (Figure 5a). It is thus possible that one of the interlocked chromosomes can be extricated by movements of its unpaired telomeres. Note in Figure 5a that the homologues of bivalent 7 pass through the unpaired region of bivalent 3 from opposite sides and thus, that this bivalent interlocking should be considered as two separate events, i.e. as two chromosome interlockings. The bivalent interlocking and three of the chromosome interlockings have occurred between the bivalents 3,4 and 7 combining them into a complex configuration (Figure 5a). The lateral component discontinuity of bivalent 2 (Figure 5c) probably results from resolution of a previous interlocking between this and bivalent 15 , as the two free ends of bivalent 2 are located in the vicinity of the synaptonemal complex of bivalent 15 .

Nucleus B contains three chromosome interlockings, one bivalent interlocking and seven chromosome breaks. Six of these breaks have occurred in chromosomes engaged in nonhomologous pairing (see section 3.4.3.) and two of them were in the proximity of pairing partner exchanges (Figure 14a), while in four cases the free ends of broken chromosomes were observed in the vicinity of putative interlockings.

Interlockings or breaks were not observed in

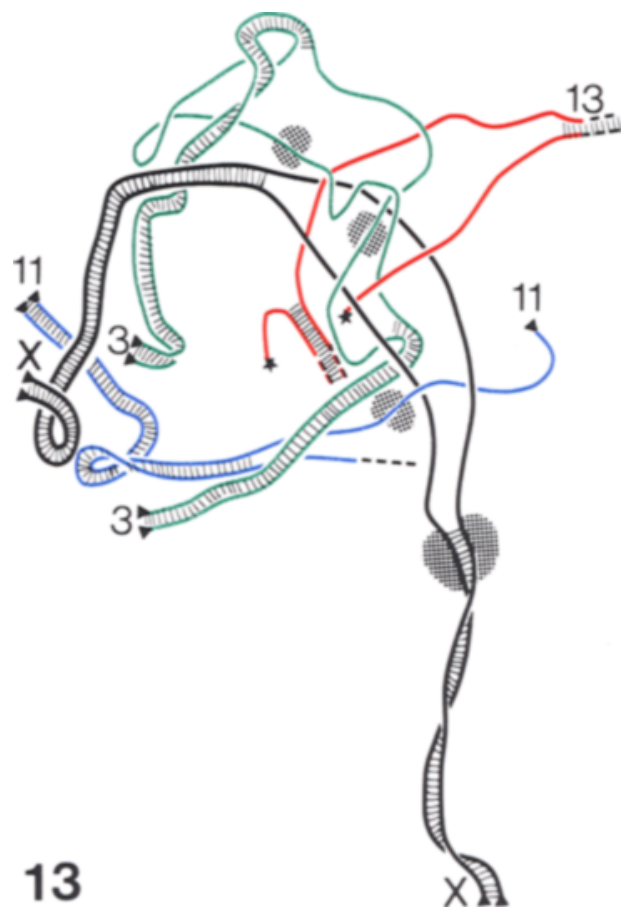

Figure 13. Reconstruction of an interlocking involving partially paired chromosomes $3,11,13$ and $X$ from a mid zygotene nucleus (Nucleus $B$ in Table V). For clarity, bivalents 11 and 13 are not shown in full length. The free ends in the broken chromosome 13 are denoted by asterisks. 
Table V.

Interlockings and breaks in two mid zygotene nuclei.

Nucleus A

Bivalent 4 is interlocked between the homologues of bivalent 3 , and one homologue of bivalent 3 is interlocked by bivalent 4 (Figure 5a).

One homologue of bivalent 3 is interlocked and the other is entrapped by the homologues of bivalent 7 (distance between the unpaired telomeres is $0.7 \mu \mathrm{m}$ ) (Figure $5 \mathrm{a}$ ).

One homologue of bivalents 17 and 2 are interlocked between the homologues of bivalent 1 (Figure $5 \mathrm{c}$ ).

One homologue of bivalent 2 is broken, the free ends being in the proximity of the synaptonemal complex of bivalent 15 (Figure 5c).

Nucleus B

Both homologues of bivalent 5 are broken (Figure 14a). Bivalent 8 passes between the broken ends.

Univalent 19 is broken and most of its short arm is nonhomologously paired with chromosome 5 (Figure 14a).

The other univalent 19 is entrapped by bivalent 13 , and one homologue of bivalent 13 is broken. The terminal segment of univalent 19 is broken and nonhomologously paired with bivalent 13 (Figure 14a).

One homologue of bivalent 9 is broken in the proximity of the pairing fork of the $\mathrm{X}$ bivalent.

One homologue of bivalent 14 is broken (Figure 14b).

Bivalent 13 and one homologue of bivalents 11 and 3 are interlocked by the homologues of the $\mathrm{X}$ bivalent (Figure 13).

One homologue of bivalent 1 is interlocked between the homologues of bivalent 3 .

the three late zygotene nuclei where pairing and synaptonemal complex formation was almost complete (Table II).

\subsubsection{Nonhomologous pairing}

Nonhomologous pairing was revealed in two of the ten serially sectioned and photographed zygotene nuclei. The synaptonemal complex combining the nonhomologously paired segments was indistinguishable from the rest of the synaptonemal complex in the nuclei. One of the nuclei could be fully reconstructed (Nucleus B in section 3.4.2.), while in the second, extensive chromosome breakage rendered the reconstruction impossible. Both nuclei came from the same ovary and were classified as being at mid zygotene. Their general appearance and ultrastructure were indistinguishable from that of normal oocytes, except that they possessed a number of well-defined recombination bars (see section 3.5). In ten additional nuclei analyzed from the same ovary (one mid, two late zygotene and 7 pachytene nuclei) only homologous pairing was revealed.

In Figure 14 are shown reconstructions and diagrams of three multiple associations from the fully reconstructed nucleus. In addition, a 0.5 $\mu \mathrm{m}$ stretch of nonhomologous pairing with a synaptonemal complex was observed between the telomeric region of the long arm of chromosome 22 and the short arm of chromosome 15. Altogether, 8 chromosome pairs, namely chromosomes 5, 8, 11, 13-15, 19 and 22 were involved in the formation of two associations of three chromosomes, one association of four and one of six chromosomes. The total length of the seven nonhomologous segments is $11.8 \mu \mathrm{m}$, which represents 3.4 percent of the total synaptonemal complex in the nucleus. The length of the individual segments ranged from 0.5 to 4.7 $\mu \mathrm{m}$.

In both associations of three chromosomes, one chromosome was broken in the vicinity of the pairing partner exchange. The breaks found in the associations of four and six chromosomes had all occurred outside the region of pairing partner exchange, three of them being in regions of putative interlockings (Table V).

In one case, the three lateral components were paired side by side by two central regions over a distance of $0.2 \mu \mathrm{m}$ in the region of pairing partner exchange (Figure 16).

Neither recombination nodules nor bars were observed at the central regions of the synaptonemal complex in the nonhomologously paired segments. 

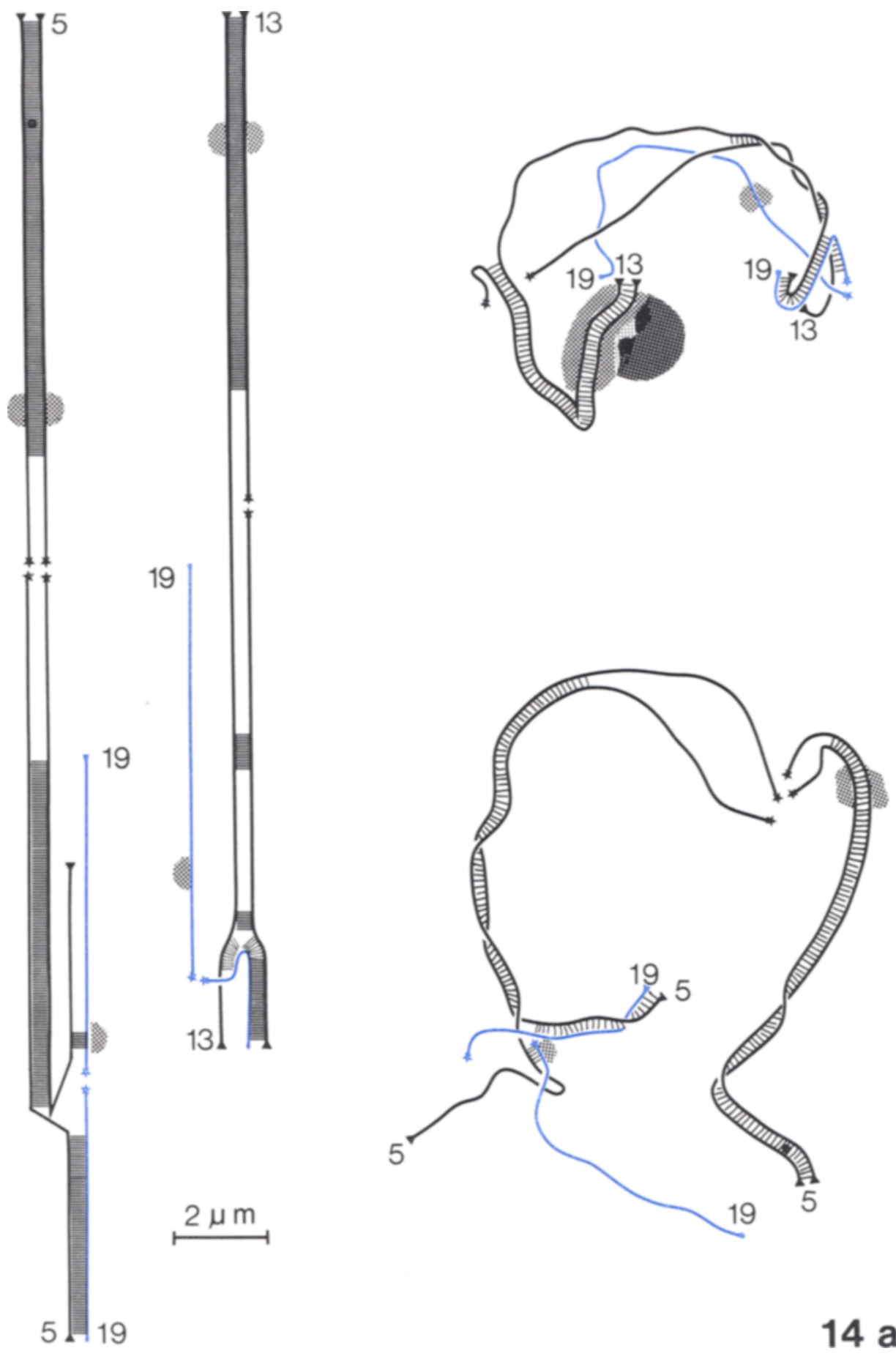

14 a 

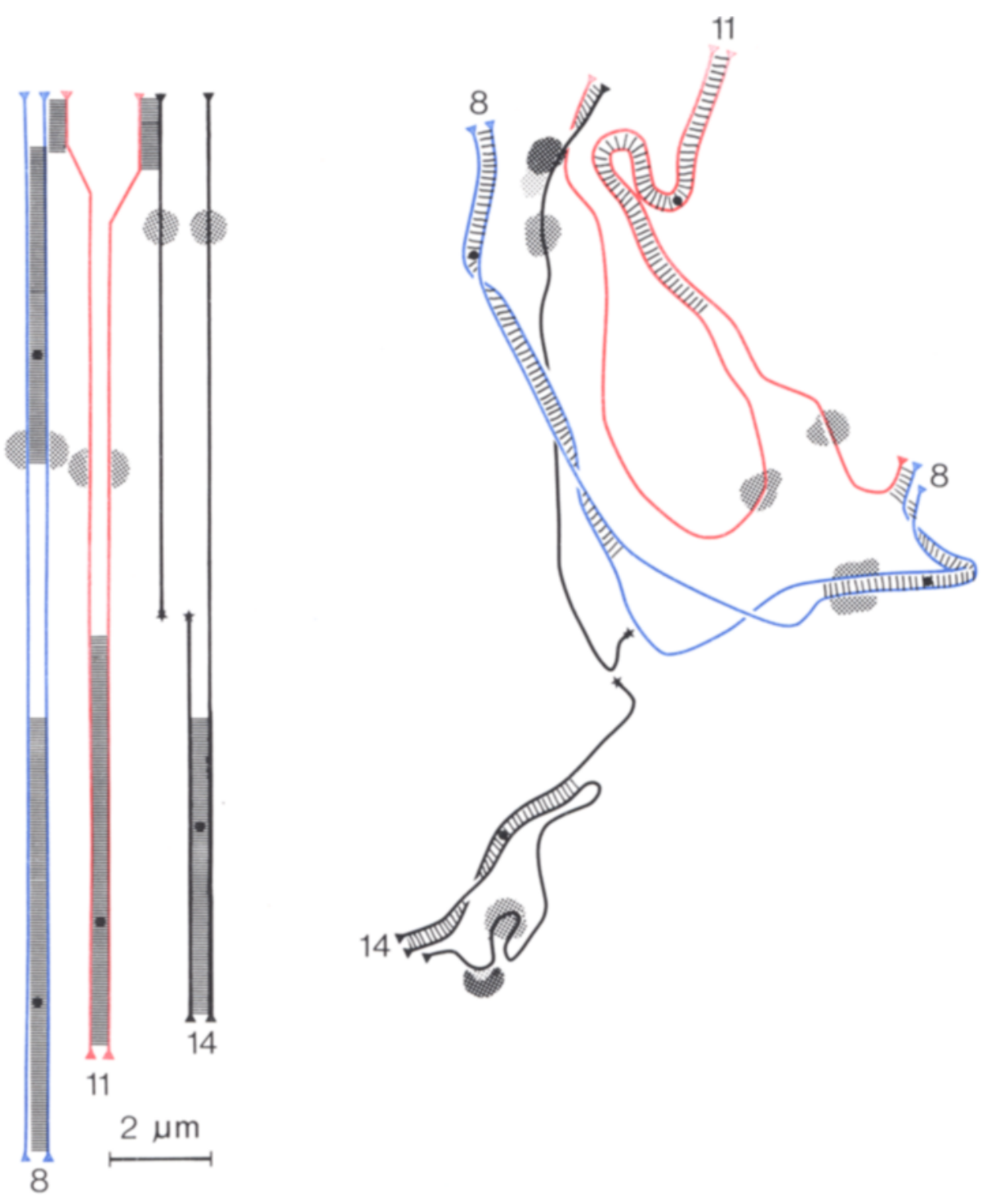

14

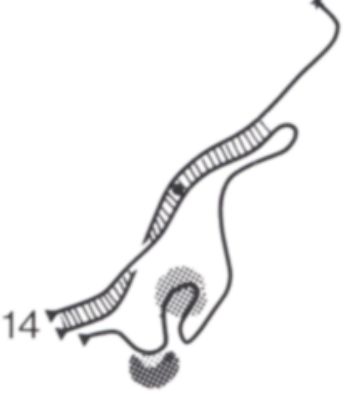

\section{$14 \mathrm{~b}$}

Figure 14. Reconstructions and diagrammatic representations of two associations of three and one association involving six chromosomes from a mid zygotene nucleus (Nucleus B in section 3.4.2.). The free ends of broken chromosomes are denoted by asterisks. Electron micrographs showing the centromere region of chromosome 19 which is nonhomologously paired with an acentric segment of chromosome 5 and the shift of pairing partners of bivalents 8 and 11 is presented in Figures 15 and 16.

\subsection{Recombination nodules and bars}

Recombination nodules were present at the central region of the synaptonemal complex of mid and late zygotene oocytes. Their attachment may coincide with the organization of the cen- tral region (Figure 17a). As shown in Figure 17, the recombination nodules displayed a wide range of shapes from globular to rod-like, the short diameter ranging from 50 to $100 \mathrm{~nm}$ and the long from 50 to $150 \mathrm{~nm}$. Generally, the nod- 


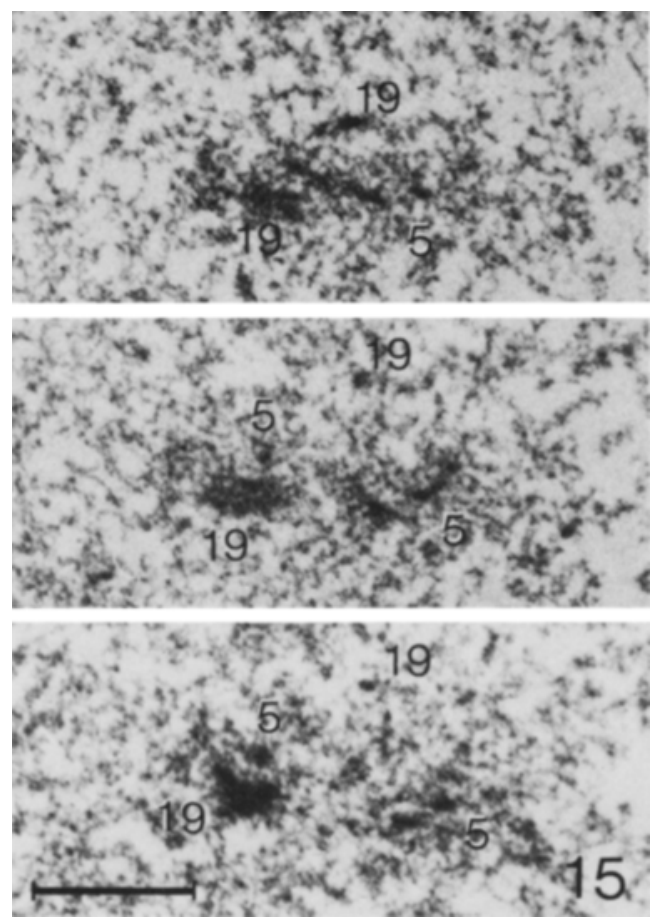

Figure 15. Three consecutive sections through the centromere region of the chromosome 19 paired nonhomologously with the chromosome 5 (see Figure 14). (Bar $=1 \mu \mathrm{m})$.

ules were connected to the lateral components by fine fibrils. In a few cases, the connections to the lateral components were as prominent as the nodule itself (Figure 17) forming a bar-like structure which bridged the lateral components at right or oblique angles to the complex. This structure is interpreted as being an intermediate between recombination nodules and recombination bars which also have been found in pachytene spermatocytes (19). One nucleus (Nucleus $B$ in section 3.4.2) differs from the others by possessing 8 intermediate and 7 well-defined recombination bars.

The total number of recombination nodules and nodule-bar intermediates per nucleus in all zygotene nuclei is given in Table II and their distribution in one early and one late zygotene nucleus in Figure 11. About 70 recombination nodules per oocyte nucleus at late zygotene $(98 \%$ pairing) compare to about 75 at early pachytene
Figure 16. Six consecutive micrographs showing a shift of pairing partners between bivalents 11 and 8 (see Figure 14). Three lateral components (both homologues of bivalent 8 and one homologue of bivalent 11) are paired with two central regions (arrows) over a distance of about $0.2 \mu \mathrm{m}$ (Figures $\mathrm{c}$ and $\mathrm{d}$ ). The additional lateral components seen in the micrographs are those of chromosome 13 and chromosome 19. Note that chromosome 19 is nonhomologously paired with chromosome 13. $(\mathrm{Bar}=1 \mu \mathrm{m})$.

of spermatocytes (28). Since chiasmata originate from recombination nodules, the potential for chiasmata formation is the same in both sexes, in spite of the presence of twofold longer synaptonemal complexes in the oocyte. A more detailed analysis of the recombination nodules and bars in the oocytes will be presented in connection with the pachytene data.

\section{DISCUSSION}

\subsection{General aspects}

The present observations on the general morphology and the temporal sequence of leptotene and zygotene oocytes are in agreement with previous ultrastructural investigations on human oocytes $(1,2,9,32)$. It should, however, be stressed that the temporal changes are not as synchronous as previously implied. In contrast to earlier reports, the results of this study have revealed considerable intracellular asynchrony in the development of the different parameters. For example, one nucleus exhibits a zygotene character of bouquet formation, a leptotene character in the development of the lateral components, while the mitochondria are arranged as seen in the oogonia, i.e. in parallel layers sepa-

Figure 17. Micrographs showing the ultrastructure of recombination nodules (RN) and intermediates between recombination nodules and bars (RB) at zygotene. Figures $17 \mathrm{a}, \mathrm{b}, \mathrm{d}$ and $\mathrm{g}$ are longitudinal sections and $17 \mathrm{c}$, e and $f$ are cross sections. The central region in Figure 17a has not yet attained its complete organization. Note the fibrillar connections between the lateral components and the recombination nodules. The micrographs in Figures $17 \mathrm{~b}$-f are printed at the same magnification. $(\operatorname{Bar}=0.5 \mu \mathrm{m})$. 

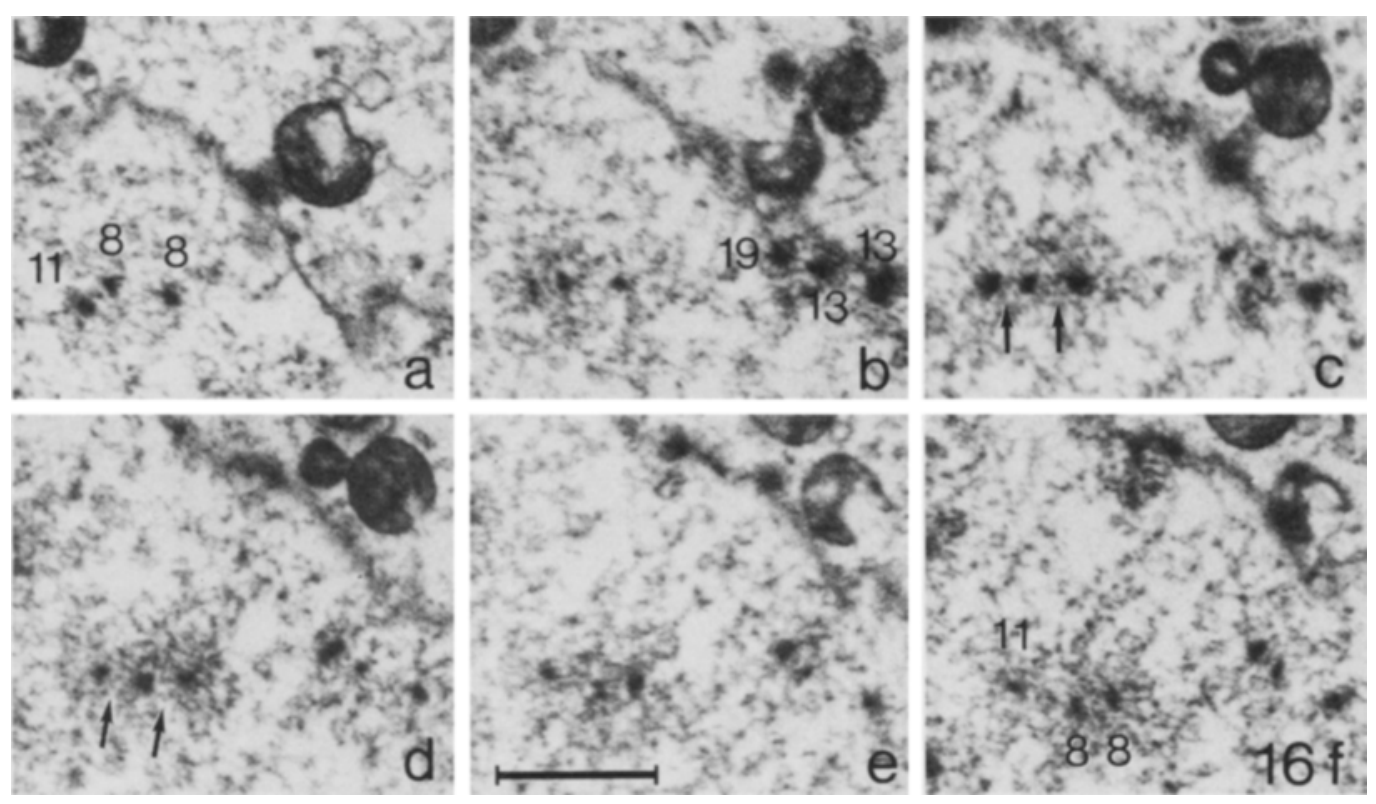

rated by double membranes (32). Striking are differences in nucleolar morphology among the oocytes at the same stage of meiosis. This asynchrony stresses that stage determination is only reliable in nuclei which are partially or completely serially sectioned.
Some of this asynchrony may reflect stages of cellular and nucleolar degeneration. Cellular degeneration of the ovary is extensive, and although all nuclei have been selected as having a normal ultrastructure, it cannot be excluded that degeneration has started in some of them.
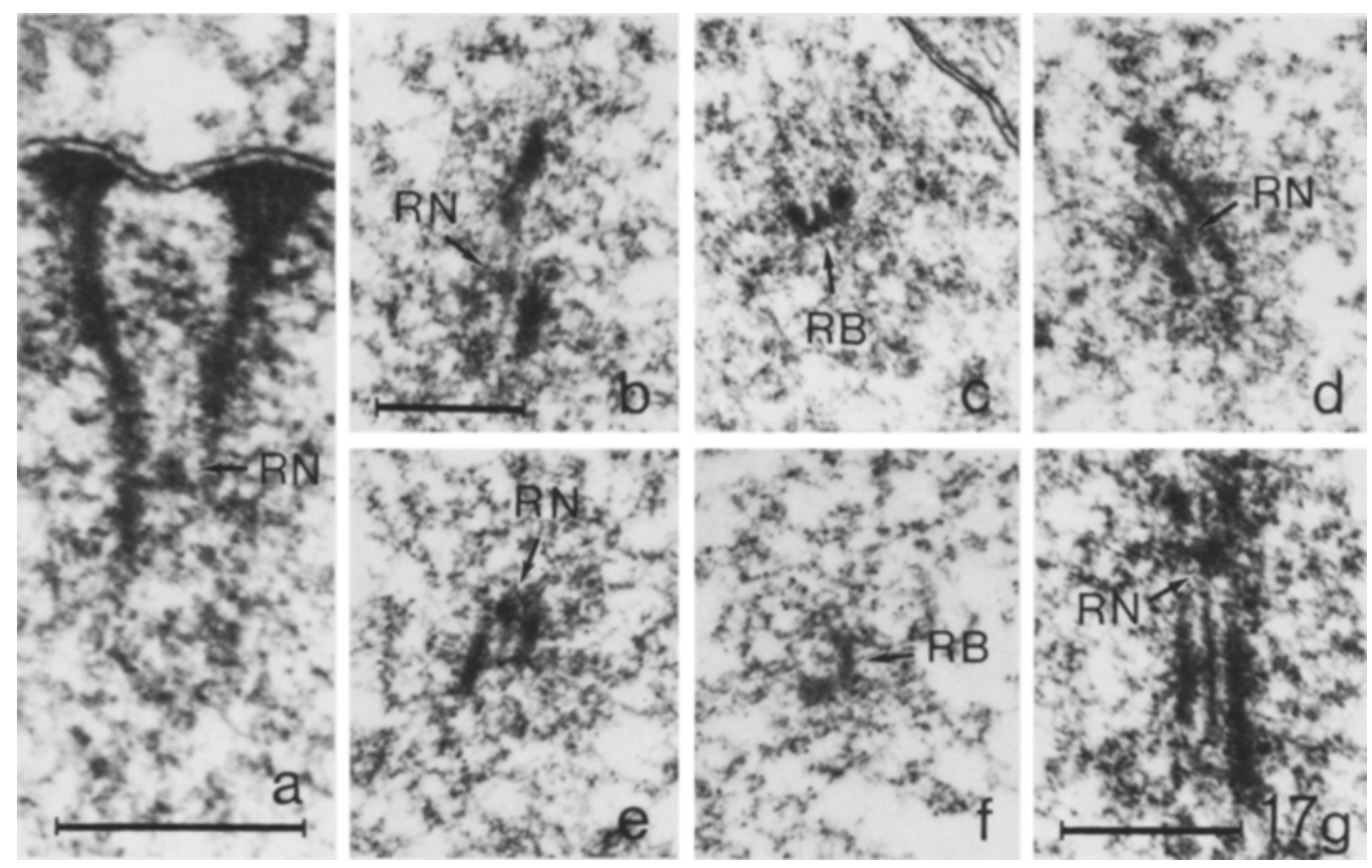


\subsection{The zygotene nucleus}

The ultrastructural characteristics of zygotene oocytes are very similar to those of spermatocytes at the same stage of development. The ultrastructure of the chromosomes, their centromere and telomere regions and the synaptonemal complex is virtually indistinguishable from that reported for the male $(13,28)$. The most conspicuous difference is the arrangement of the mitochondria in a concentric layer around the nuclear envelope in the oocytes. Likewise, the fine structure of the nucleoli as well as the number of acrocentric chromosomes associated with nucleoli are different from that of the spermatocytes. At late zygotene, virtually all acrocentric chromosomes in spermatocytes are associated with a nucleolus through a spherical, fibrillar body. All nucleoli contain a reticulum of electron transparent cavities, while spherical, compact nucleoli with dark bodies first appear from the end of mid pachytene (18). In the oocytes, a variety of different shaped nucleoli are present at the same substage attached to a fewer number of acrocentric chromosomes than in the spermatocytes.

Three to seven nucleoli (Table I) are present in the analyzed zygotene nuclei. The observation by STAHL and LuCIani (31) using the light microscope that 62.5 percent of leptotene and 83 percent of zygotene oocytes contain only one nucleolus and that more than three nucleoli are observed in only 0.9 percent of the leptotene oocytes and never at zygotene is at variance with the present investigation and may reflect the insufficient resolution by the light microscope techniques.

\subsection{The zygotene karyotype}

The total lateral component length of one genome in the zygotene oocytes amounts to $473 \pm$ $57 \mu \mathrm{m}$ and is approximately twice as large as the value $235 \pm 26 \mu \mathrm{m}$ (mean of ten zygotene nuclei) reported for the male (28). This unequivocally shows that the lateral component length of a given chromosome can not only be a function of its DNA length.

Apart from this difference, the relative lengths and centromere indices of the autosomal bivalents correspond with those of spermatocytes (Table III). This shows that the dif- ference in contraction of meiotic chromosomes in spermatocytes and oocytes is not confined to specific chromosomes or chromosome arms but affects all chomosomes in a similar manner. Furthermore, as the markers on bivalents 1 and 6 have the same relative position in the two sexes, all chromosome segments are probably affected in the same way. However, sex-specific chromomeric variation has been reported (5) for the mouse and human pachytene maps. This may either signify differential contraction of individual chromosome segments in the two sexes, or a different pattern of chromatin condensation.

Absolute differences in length between the same bivalents from different oocytes are evident from Table III and Figure 11 and appear to exceed the differences accountable for by errors of the method, estimated to be approximately $10 \%$ of the measured lengths. This is also the case in human spermatocytes (13) showing that the length of the bivalent can to some extent be controlled by the individual cell.

As pointed out in section 3.3, the X chromosome appears morphologically indistinguishable from the autosomes. This is also the case at late zygotene in the spermatocytes, whereas after pairing with the $Y$ chromosome and the formation of the XY body both chromosomes undergo structural modifications (18).

\subsection{Chromosome pairing}

The general scheme of chromosome pairing and synaptonemal complex formation observed in human oocytes is in agreement with that reported for human spermatocytes (28) and can be summarized as follows. At leptotene, segments of lateral components are organized in several regions of the chromosomes, often including the telomere regions. The telomeres become attached to the nuclear envelope, the attachment sites being distributed more or less evenly throughout the inner membrane. Subsequently, at early zygotene the telomeres congregate to a limited area of the nuclear envelope by a floating of the attachment sites within the membrane, a movement which in the human may be mediated by the cytoplasmic spheres connected to the telomeres. In most cases, pairing and synaptonemal complex formation start in the vicinity of the telomeres, but also interstitial pairing, which 
is rare in human spermatocytes occurs quite frequently in human oocytes. This difference may be related to the different chromosome lengths in the two sexes. In meiocytes with long synaptonemal complexes probably more opportunity is available for random meeting of homologous chromosome segments and consequently synapsis of interstitial segments is more frequent. Exclusive initiation of pairing from the telomeres is generally favoured in organisms with short chromosomes such as Bombyx males and females $(15,26)$ and Coprinus (17), while simultaneous initiation of pairing at telomeres and interstitially is characteristic for organisms with long chromosomes such as Lilium, maize and wheat $(7,11,12)$. Interstitial initiation has however, been reported in Sordaria (35), which has few and short chromosomes, suggesting that also the number of chromosomes may influence the pairing kinetics. Finally, it should be noted that interstitial pairing is often observed in segments where chromosome movements appear to be impeded by interlocking $(26,28)$.

The present investigation has shown that interlocking of chromosomes and bivalents occurs regularly during chromosome pairing in human oocytes. This is also the case in spermatocytes (28) as well as in all other organisms where complete reconstructions of zygotene nuclei have been performed, i.e. Bombyx males (15) and females (26), Coprinus (17), Triticum (11) and Sordaria (36).

The absence of interlockings in the three almost completely paired zygotene nuclei furthermore shows that the interlockings are resolved by the end of zygotene. The observation that chromosome and bivalent breakages are common at mid zygotene but absent at late zygotene and that the broken ends of the lateral components in many cases are located in regions of putative interlockings, gives additional support to the proposal that resolution of interlockings occurs by breakage and reunion of the interlocked chromosomes (see 16 and 29 for review).

The frequency of interlockings and breaks in the two human oocyte nuclei is 5.7 times higher than that reported for human spermatocytes, where the mean frequency of breaks and interlockings was reported to be 1.5 per nucleus (28). It is conceivable that the higher level of aberra- tions in the female can be attributed to the longer complement of the female chromosomes contained within a nucleus of approximately the same volume as that of the spermatocyte.

The rather high frequency of nonhomologous pairing by synaptonemal complex in two mid zygotene oocyte nuclei is unexpected. It has been shown in several organisms (e.g. $11,17,27$ ), as well as the human male (14) that zygotene pairing is strictly limited to homologous segments, while subsequently unpaired chromosomes or chromosome segments can associate non-homologously by a synaptonemal complex. Nonhomologous pairing has been found in the following two situations:

a) When the possibility for homologous association is lacking or exhausted as is the case in haploids (6), triploids (27) and organisms heterozygous for structural rearrangements.

b) In defiance of an opportunity for homologous or homoeologous synapsis. Short stretches of nonhomologous pairing are occasionally observed in diploids in segments where the pairing appeared to be mechanically impeded by interlockings or chromosome breaks (e.g. 12, 17), HoBOLTH (11) reports on more extensive, apparently nonhomologous pairing at zygotene in allohexaploid wheat, which may be attributed to the fact that wheat is an amphidiploid in which initial homoeologous pairing between chromosomes of different origin may proceed into adjacent, nonhomologous regions.

The situation revealed in the two mid zygotene oocytes may be a result of the extensive interlockings or it may be related to the two phase pairing recognized in triploid Bombyx females (27), assuming that in the segments in question the pairing was delayed beyond the stage where specificity of the regions to be paired is mandatory (16). The partially synapsed chromosomes will then pair to the nearest neighbour, despite the lack of homology. This would imply that at least some chromosome segments in the two nuclei represent pachytene conditions despite the incomplete chromosome pairing. This would constitute an additional indication of extreme intranuclear asynchrony. This proposal also explains the presence of well-defined recombination bars, which in human spermatocytes were not observed until pachytene. 


\section{ACKNOWLEDGEMENTS}

I would like to express my gratitude to Professor Diter von Wettstein, Dr. Preben B. Holm and Dr. Søren W. Rasmussen, who suggested the investigation and also greatly influenced this work through inspiring scientific and practical guidance. Their valuable critisism of this communication is also highly appreciated. I am also indebted to Professor Frans Bierring, Dr. Mogens RønNe, Dr. Jørgen GRINSTED and ULLA HAUSCHILD for their assistance in obtaining the ovaries, and it is a pleasure to thank JEAN SaGe, Ann-Sofi Steinholtz and Dan Olsson for their outstanding technical assistance.

This work was financially supported by grant 202-76-1 BIO DK and BIO-E-417-DK(G) from the Commission of the European Communities to Professor D. von WetTstein.

\section{REFERENCES}

1. BAKER, T.G.: A quantitative and cytological study of germ cells in human ovaries. Proc. Roy. Soc. B. 158, 417-433 (1963)

2. Baker, T.G. \& L.L. Franchi: The fine structure of oogonia and oocytes in human ovaries. J. Cell. Sci. 2, 213-238 (1967)

3. Berthelsen, J.G., P.B. Holm \& S.W. RASMUSSEN: Three ultrastructural markers on pachytene bivalents of human spermatocytes. Carlsberg Res. Commun. 45, 25-28 (1980)

4. Carr, D.H. \& M. Gedeon: Population cytogenetics of human abortuses. In: Population cytogenetics. Studies in Humans. (E.B. Hook \& I.H. Porter eds.) Acad. Press, Inc., New York, pp. 1-9 (1977)

5. FANG, J.-S. \& G. JAGIELLO: A pachytene map of the mouse spermatocyte. Chromosoma (Berl.) 82, 437-445 (1981)

6. Gillies, C.B.: The nature and extent of synaptonemal complex formation in haploid barley. Chromosoma (Berl.) 48, 441-453 (1974)

7. Gillies, C.B.: An ultrastructural analysis of chromosomal pairing in maize. C. R. Trav. Lab. Carlsberg 40, 135-161 (1975)

8. Gillies, C.B.: Synaptonemal complex and chromosome structure. Annu. Rev. Genet. 9, 91-109(1975)

9. Gondos, B., P. Bhiraleus \& C.J. Hobel: Ultrastructural observations on germ cells in human fetal ovaries. Amer. J. Obstet. Gynec. 110, 644652 (1971)

10. Gondos, B. \& C.J. Hobel: Germ cells degeneration and phagocytosis in the human foetal ov- ary. In: The development and maturation of the ovary and its functions. (H. Peters ed.) Internat. Congress Ser. 267 pp.77-83 (1973)

11. Новоцтн, P.: Chromosome pairing in allohexaploid wheat var. Chinese Spring. Transformation of multivalents into bivalents, a mechanism for exclusive bivalent formation. Carlsberg Res. Commun. 46, 129-173 (1981)

12. HoLM, P.B.: Three-dimensional reconstruction of chromosome pairing during the zygotene stage of meiosis in Lilium longiflorum (Thunb.). Carlsberg Res. Commun. 42, 103-151 (1977)

13. Holm, P.B. \& S.W. Rasmussen: Human meiosis I. The human pachytene karyotype analyzed by three dimensional reconstruction of the synaptonemal complex. Carlsberg Res. Commun. 42, 283-323 (1977)

14. Holm, P.B. \& S.W. Rasmussen: Human meiosis III. Electron microscopical analysis of chromosome pairing in an individual with a balanced translocation $46, \quad X Y, t(5 p-; 22 p+)$. Carlsberg Res. Commun. 43, 329-350 (1978)

15. Holm, P.B. \& S.W. Rasmussen: Chromosome pairing, recombination nodules and chiasma formation in diploid Bombyx males. Carlsberg Res. Commun. 45, 483-458 (1980)

16. Holm, P.B. \& S.W. Rasmussen: Chromsome pairing, crossing over, chiasma formation and disjunction as revealed by three dimensional reconstructions. In: International Cell Biology 1980-1981. (H.G. Schweiger ed.) Springer-Verlag, Berlin, Heidelberg \& New York, pp.194204 (1981)

17. Holm, P.B., S.W. RasmuSSEN, D. ZICKLER, B.C. Lu \& J. SAGE: Chromosome pairing, recombination nodules and chiasma formation in the basidiomycete Coprinus cinereus. Carlsberg Res. Commun. 46, 305-346 (1981)

18. Holm, P.B. \& S.W. Rasmussen: Human meiosis V. Substages of pachytene in human spermatocytes. Carlsberg Res. Commun. 351383 (1983)

19. Holm, P.B. \& S.W. Rasmussen: Human meiosis VI. Crossing over in human spermatocytes. Carlsberg Res. Commun. 385-413 (1983)

20. Holm, P.B. \& S.W. Rasmussen: Human meiosis VII. Chiasma formation in human spermatocytes. Carlsberg Res. Commun. 415456 (1983)

21. Manotaya, T. \& E.L. Potter: Oocytes in prophase of meiosis from squash preparations of human fetal ovaries. Fertil. Steril. 14, 378-392 (1963) 
22. Moses, M.J.: Synaptinemal complex. Annu. Rev. Genet. 2, 363-412 (1968)

23. Polani, P.E.: Chiasmata, Downs syndrome and nondisjunction. An overview. In: Trisomy 21 (Downs syndrome). Research perspectives. (F.F. de la Cruz and P.S. Gerald eds.) University Park Press, Baltimore, pp. 111-130 (1980)

24. Paris Conference: Standardization in human cytogenetics. Cytogenetics 11, 313-362 (1972)

25. Parvinen, M. \& K.O. Söderström: Chromosome rotation and formation of synapsis. Nature 260, 534-535 (1976)

26. Rasmussen, S.W.: The meiotic prophase in Bombyx mori females analyzed by three dimensional reconstruction of synaptonemal complexes. Chromosoma (Berl.) 54, 245-293 (1976)

27. Rasmussen, S.W.: Chromosome pairing in triploid females of Bomyx mori analyzed by three dimensional reconstructions of synaptonemal complexes. Carlsberg Res. Commun. 42, 163197 (1977)

28. Rasmussen, S.W. \& P.B. Holm: Human meiosis II. Chromosome pairing and recombination nodules in human spermatocytes. Carlsberg Res. Commun. 43, 275-327 (1978)

29. Rasmussen, S.W. \& P.B. Holm: Mechanics of meiosis. Hereditas 93, 187-216 (1980)
30. Sotelo, R.: Ultrastructure of the chromosomes at meiosis. In: Handbook of molecular cytology. (A. Lima-de-Faria ed.) North-Holland Publ. House, Amsterdam, pp. 412-433 (1969)

31. Stahl, A. \& J.M. Luciani: Nucleoli and chromosomes: Their relationships during the meiotic prophase of the human fetal oocyte. Humangenetik 14, 269-284 (1972)

32. Stegner, H.E. \& H. Wartenberg: Elektronmikroskopische Untersuchungen an Eizellen des Menschen in verschiedenen Stadien der Oogenese. Arch. Gynäk. 199, 151-172 (1963)

33. WestergaARD, M. \& D. von Wettstein: The synaptinemal complex. Annu. Rev. Genet. 6, 71-110 (1972)

34. Witschi, E.: Embryology of the ovary. In: The ovary. (H.G. Grady \& D.E. Smith eds.) Williams \& Wilkins, Baltimore, pp.1-10 (1963)

35. ZICKLER, D.: Development of the synaptonemal complex and the "recombination nodules" during meiotic prophase in the seven bivalents of the fungus Sordaria macrospora Auersw. Chromosoma (Berl.) 61, 289-316 (1977)

36. ZiCKLER, D. \& J. SAGE: Synaptonemal complexes with modified lateral elements in Sordaria humana: development of and relationship to the "recombination nodules". Chromosoma (Berl.) 84, 305-316 (1981) 